\title{
Protracted development of visuo-proprioceptive integration for uni-and bimanual motor coordination
}

\author{
Marie Martel ${ }^{1,2}$, Jose P. Ossandón ${ }^{3}$, Boukje Habets ${ }^{1,2}$, \& Tobias Heed $^{1,2}$ \\ ${ }^{1}$ Biopsychology \& Cognitive Neuroscience, Faculty of Psychology \& Sports Science, Bielefeld \\ University, Bielefeld, Germany \\ ${ }^{2}$ Center of Excellence in Cognitive Interaction Technology (CITEC), Bielefeld University, Bielefeld, \\ Germany \\ ${ }^{3}$ Biological Psychology and Neuropsychology, Faculty of Psychology and Human Movement Science, \\ University of Hamburg, Hamburg, Germany
}

Running title ( 40 chars of 40 allowed): Development of uni- and bimanual coordination

\section{ACKNOWLEDGEMENTS}

This work was supported by the German Research Foundation (DFG) through the Cluster of Excellence Cognitive Interaction Technology 'CITEC' at Bielefeld University, an Emmy Noether grant to TH (He 6368/1-1, 1-2, 1-3), and by the Collaborative Research Center SFB936, project B1. We thank all children who participated in this study, and their parents. We thank Valerie Gockel, Alexander Seriyo and Frederick Thiemer for their support with data acquisition, Martin Bertele and Dennis Pauer for programming assistance, and Luke Miller, Yannish Ramgulam, Christian Seegelke, and Kenan Suljic for support with data analysis.

\section{CONFLICTS OF INTEREST}

There are no conflicts of interest. 


\section{ABSTRACT}

Motor coordination, either between two limbs or between a limb and another sensory signal, depends on the interplay between motor control and sensory processing. Although profusely studied in relative isolation, knowledge of the interdependence between different control mechanisms and sensory sources is incomplete. We addressed this gap with a cross-sectional, developmental approach. Children aged 4 to 12 years $(\mathrm{N}=120)$ performed multiple versions of a hand-circling task. We show that coordination of symmetric bimanual movements is an independent mechanism that develops first, followed by proprioceptive and visual unimanual guidance. The ability to strategically select some, but ignore other, sensory information for asynchronous bimanual motor coordination develops last. Partial correlations between different tasks suggest interdependent, chained development within individuals. These results suggests that bimanual coordination is not a subcategory of general coordination ability, but is based on independent, likely motor-based, factors. Moreover, the development of different motor functions is serially interdependent.

\section{KEYWORDS}

Proprioception; feedback; multisensory integration; sensorimotor processing; vision; Lissajous 


\section{1 | INTRODUCTION}

Motor development protracts over almost two decades before humans possess the full set of abilities of adults. Mastering a new motor skill often sets the stage for learning further novel skills and changes the way the child interacts with its environment (Adolph \& Franchak, 2017; Adolph \& Hoch, 2019; Hamilton, Southgate, \& Hill, 2016). The long development of motor control is mirrored by similarly protracted development of (multi-)sensory processing. For instance, adult-like integration of multisensory input is usually not observed until at least 8-10 years of age (for review, see, Burr \& Gori, 2012; Gori, 2015), and proprioceptive processing appears to undergo changes until at least the age of 15 (Assaiante, Barlaam, Cignetti, \& Vaugoyeau, 2014). Current models of motor control propose that motor planning and execution indispensably rely on sensory information about body and environment, as well as on internal models that predict the sensory consequences of planned and ongoing movement based on an efference copy, that is, a copy of the initiated movement command that is provided to the internal model. Comparison of predictions with sensory feedback allows to correct movement online (Shadmehr \& Krakauer, 2008). However, how motor control and its relationship to sensory processing develop remains poorly understood. Most previous studies have focused on one or few sensorimotor functions, have used a diverse range of methods and paradigms, and have often studied only a narrow age range.

We tested seven variations of an established circling paradigm with 4 to 12 -year-old children and young adults. Participants had to coordinate one or both hands while visual and proprioceptive information was, or was not, available. This design addressed three related functional aspects of motor control: actively coordinating two body parts symmetrically (bimanual symmetry tasks), synchronizing a single body part with either passive-proprioceptive or visual sensory signals (unimanual symmetry tasks), and coordinating body parts when visual implied symmetry only when the hands moved asymmetrically, requiring asymmetric motor commands and resulting in asymmetric proprioceptive feedback (bimanual asymmetry task). In the unimanual symmetry tasks, one hand is still (or moved passively), and so no efference copy is produced for that hand. In the bimanual asymmetry task, the efference copies produced for the two hands are asymmetrical and, therefore, counterproductive for coordination. In both tasks participants must, thus, rely only on sensory feedback, either visual or proprioceptive. By testing all tasks in the same participants, we discriminated between different components of motor control, their reliance on sensory information, and their cross-sectional development.

To coordinate two body parts, participants might simply dispatch parallel movement commands to the two limbs, rather than relying on sensory feedback. Indeed, the motor system 
prefers symmetrical over other types of bimanual movements (e.g. Brandes, Rezvani, \& Heed, 2017; Kelso, Scholz, \& Schöner, 1986; Spencer, Ivry, Cattaert, \& Semjen, 2005). Many studies have contrasted symmetrical with parallel movements in hand circling tasks. Here, symmetrical coordination refers to both hands circling inward (or outward), that is, in opposite directions, and parallel coordination refers to both hands circling in the same direction. Performance of symmetrical movements is regularly better than that of parallel movements, both in circling tasks, and in other types of coordination such as finger wiggling (for review, Gooijers \& Swinnen, 2014). These findings were initially interpreted to indicate that bimanual coordination relies purely on synchronized motor commands (Cohen, 1971; Kelso, 1984; Riek \& Woolley, 2005). However, later studies also demonstrated a role of perceptual factors (for review, see Shea, Buchanan, \& Kennedy, 2016). For instance, when the index fingers are wiggled with one hand turned up, and the other down, symmetrical movements are still performed more successfully than parallel ones, although they now require concurrent activation of non-homologous muscles (Brandes et al., 2017; Heed \& Röder, 2014; Mechsner, Kerzel, Knoblich, \& Prinz, 2001).

Here, we addressed the development of bimanual coordination using the bimanual circling approach. Preference for in-phase coordination in bimanual circling tasks has been observed beginning at age 5 (Robertson, 2001), with performance improving up to adulthood (de Boer, Peper, \& Beek, 2012). Continuing from these findings, we asked whether bimanual coordination is more successful when vision of the hand movement is available than when it is not.

The second aspect of motor control of our study pertains to the coordination of a single body part with a sensory signal. Humans coordinate unimanual movements well with signals of different modalities such as vision (e.g. Buekers, Bogaerts, Swinnen, \& Helsen, 2000; Hove, Spivey, \& Krumhansl, 2010) and proprioception (e.g. Kelso, Fink, DeLaplain, \& Carson, 2001; Wing, Doumas, \& Welchman, 2010). This observation has led to the suggestion that movement coordination is perhaps entirely perceptually driven (Bingham, 2004). In this view, bimanual coordination is but a subcategory of a more general ability to coordinate by means of perception. Under this assumption, uni- and bimanual coordination should show similar developmental trajectories. If, in contrast, bimanual coordination relied predominately on motor synchronization, then one might expect different developmental trajectories for the two tasks.

Many studies that have investigated the use of sensory information for motor control (e.g. Kagerer \& Clark, 2014; P. H. Wilson \& Hyde, 2013) have uncovered extended development up to the age of at least 10 years. These previous results let us expect that unimanual coordination, too, might develop until at least this age. To test whether these potential trajectories differ across sensory 
modalities, we assessed children's ability to coordinate their hand with an externally produced visual or passive-proprioceptive signal.

The third aspect of our study pertains to situations in which movement is neither symmetrical or parallel; such coordination is very hard to perform (Kelso, 1984; Mechsner et al., 2001). Critically, transforming visual information to make it appear symmetrical can vastly improve asymmetrical coordination performance in adults. For instance, circling the hands with a $90^{\circ}$ phase difference, that is, with a relative delay of a quarter circle, is much easier when the visual feedback of the lagging hand is rotated forward by $90^{\circ}$, making the two visual signals appear in-phase (Tomatsu \& Ohtsuki, 2005). Similarly, performance improves when hand positions are merged in a Lissajous display, that is, displayed as $x$ and y coordinates of a single cursor feedback (Chiou \& Chang, 2016; Kovacs, Buchanan, \& Shea, 2009). These visualizations presumably support motor coordination by making the motor constraints perceptually identifiable or salient (Bingham, 2004).

Like unimanual coordination, such a bimanual asymmetry task requires coordination with a signal that does not, at least not directly, reflect limb position. However, differing from unimanual coordination, participants must ignore non-visual information. This is because only transformed visual information contains the correct phase information required to solve the task, whereas proprioceptive information for the two hands differs from moment to moment by task design.

Out-of-phase bimanual paradigms can also be realized with a circling paradigm in which participants must turn two handles a different number of times, such as four turns with the left for every three turns of the right hand. This is often impossible for adults to perform with veridical visual feedback but easily accomplished with transformed visual feedback that translates one circle of each hand to one-fourth or one-third of a revolution of two visual markers, thus making them to move in synchrony when participants follow the required (here, 4:3) pattern (Mechsner et al., 2001).

It is currently unknown how the ability to selective use of visual information required for this task develops. Children aged 8-10 successfully performed 2:1 wrist movements under visual guidance of a Lissajous display, even if less efficiently than adults (Leinen et al., 2016). Here, we tested a more difficult 2:3 coordination task, as well as its retention (no visual transformed feedback after some training with the transformed feedback).

In sum, the present study addressed the development of three aspects of motor control: visual and proprioceptive coordination of active bimanual control, unimanual coordination with an external visual or passive-proprioceptive guiding signal, and the ability to focus entirely on visual 
information while ignoring proprioception, to perform otherwise impossible movements. We crosssectionally investigated an extended age range of 4-12-year-old children.

\section{2 | RESULTS}

We employed a common experimental paradigm across seven tasks, illustrated in Fig. 1. Together, they address the three aspects of motor control we laid out in the introduction.

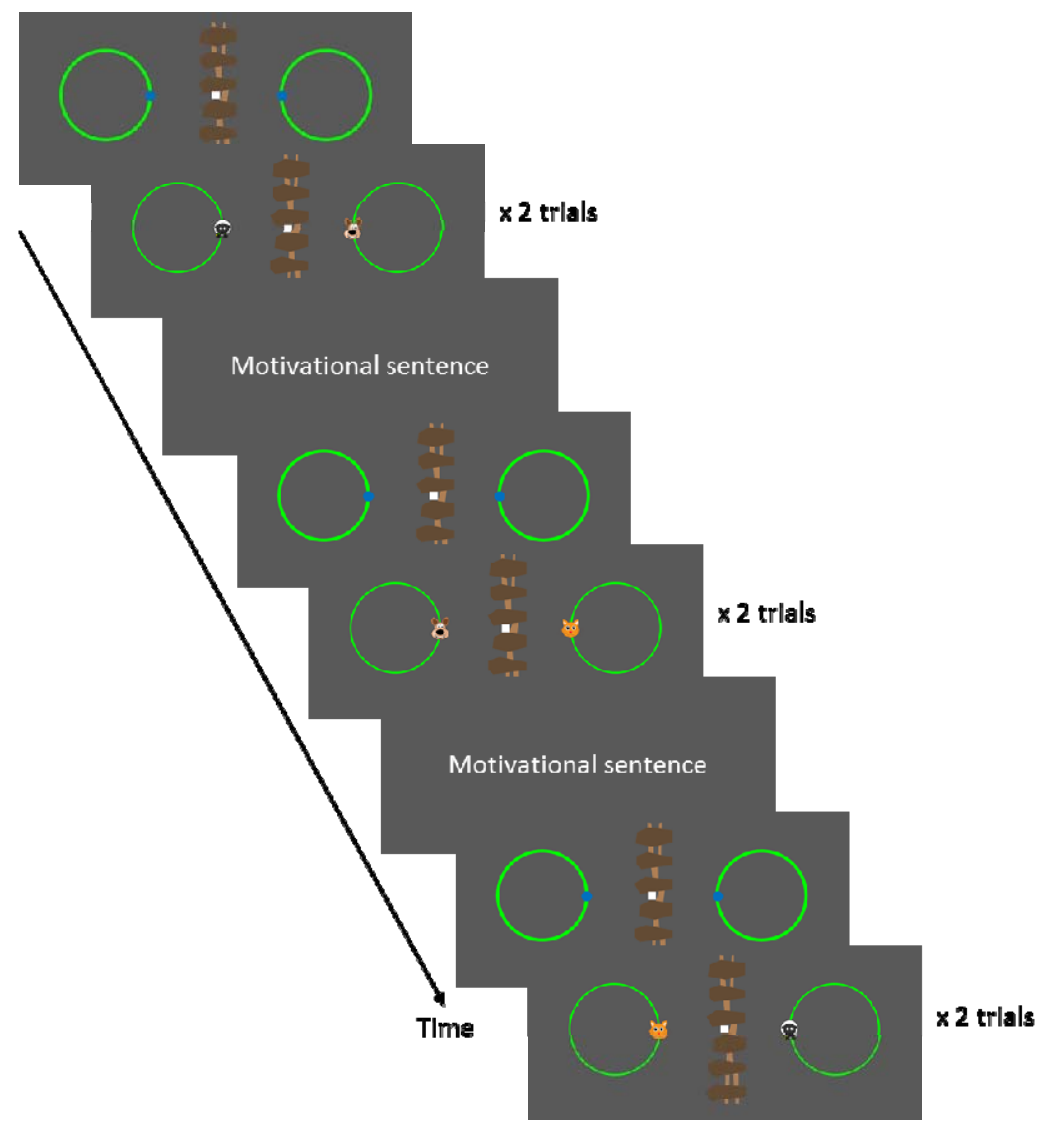

Fig. 1

General layout of the circling tasks. The display was mounted above the hands, and visual stimuli appeared as if they were at the level of the hands. During trials, movement was restricted to $10 \mathrm{~cm}$ diameter circles that were displayed on the screen. When the participant moved to the blue starting positions, a beep indicated the beginning of the trial. Participant then made continuous circling movements until a beep indicated the trial's end. Hand position was indicated by small characters, here a fox and a sheep. The task was framed using these characters; for instance, one animal was to try and catch the other. Every two trials, short motivational sentences were displayed, and the experimenter reminded the participant to fixate the white central square. 


\section{1 | Bimanual motor coordination}

\subsection{1 | Unsigned phase difference}

\subsubsection{1 | Unsigned phase difference between the hands across all ages}

We assessed the variability of children's coordination performance in a model that predicted the unsigned phase difference between the two hands with respect to the four bimanual and unimanual coordination tasks, and with respect to participants' age (see Methods for detailed description of the tasks and analysis strategy). We first describe the effects of age in the two bimanual tasks, BimanNoVis and Biman-Vis, and return to the unimanual tasks in section 2.2.1.1. In Biman-NoVis, participants had to symmetrically coordinate their unseen hands; in Biman-Vis, the same task was performed with visual feedback about hand position. Figure 2 shows that the performance in both Biman-NoVis and Biman-Vis improved with Age (left panel, dark and light red symbols). In the model, both slope coefficients were negative and different from 0 (Biman-NoVis: $\beta_{\text {Age }}=-8.8, \mathrm{HDI}_{95 \%}=[-10.9$; 7.0]; Biman-Vis: $\left.\beta_{\text {Age }}=-7.8, \mathrm{HDl}_{95 \%}=[-9.9 ;-5.7]\right)$. These negative slope values indicate that variability between the hands decreased by the respective amount of degrees with each standardized age unit of 30.3 months. The contrast between the two bimanual tasks intercept and the Task*Age interaction, reflecting differences of the slopes across tasks, were not credibly different from 0 (see Table 1 for a summary of all model estimates), thus indicating that the performance in the two bimanual tasks did not differ.

Table 1. Beta Estimates reflecting the comparison between Tasks 1 to 4 . Negative $\beta$ Estimates indicate that performance was better with age (smaller variability for each standardized unit of Age of 30.3 months). For Task, positive $\beta$ Estimates indicate that Intercept was higher in the first task of each line. Credible differences are indicated in bold and italic (HDI outside [-4.1; 4.1]). ESS stands for the Effective Sample Size as calculated during Bayesian model fitting. The Lower and Upper Confidence Interval (Cl) values constitute the 95\% High Density Interval (HDI).

\begin{tabular}{|c|c|c|c|c|c|}
\hline Effect & Contrasts & $\begin{array}{c}\boldsymbol{\beta} \\
\text { Estim } \\
\text { ate }\end{array}$ & $\begin{array}{c}\text { Lower } \\
\mathrm{Cl}\end{array}$ & $\begin{array}{c}\text { Upper } \\
\mathrm{Cl}\end{array}$ & ESS \\
\hline \multirow{4}{*}{ Age } & Biman-NoVis & -8.8 & -10.9 & -7.0 & 3961 \\
\hline & Biman-Vis & -7.8 & -9.9 & -5.7 & 4386 \\
\hline & Uniman-Vis & -21.8 & -23.7 & -19.7 & 4262 \\
\hline & Uniman-Prop & -19.5 & -21.8 & -17.1 & 5085 \\
\hline \multirow{6}{*}{ Task } & Biman-Vis - Biman-NoVis & -4.9 & -9.2 & -0.7 & 17248 \\
\hline & Uniman-Vis - Biman-NoVis & 75.3 & 71.4 & 79.1 & 16774 \\
\hline & Uniman-Vis - Biman-Vis & 80.2 & 76.5 & 84.0 & 52678 \\
\hline & Uniman-Prop-Uniman-Vis & -12.1 & -17.9 & -5.8 & 28614 \\
\hline & Uniman-Prop-Biman-NoVis & 63.2 & 56.8 & 69.5 & 17968 \\
\hline & Uniman-Prop - Biman-Vis & 68.1 & 61.8 & 74.4 & 32170 \\
\hline
\end{tabular}




\begin{tabular}{||l|l|c|c|c|c|}
\hline \multirow{4}{*}{$\begin{array}{c}\text { Interaction Task } \\
*\end{array}$} & Biman-Vis - Biman-NoVis & 1.2 & -0.00 & 2.4 & 17291 \\
\cline { 2 - 6 } & Uniman-Vis-Biman-NoVis & $\mathbf{- 1 2 . 7}$ & $\mathbf{- 1 3 . 9}$ & $\mathbf{- 1 1 . 8}$ & $\mathbf{1 6 7 1 3}$ \\
\cline { 2 - 6 } & Uniman-Vis-Biman-Vis & $\mathbf{- 1 4 . 0}$ & $\mathbf{- 1 5 . 1}$ & $\mathbf{- 1 3 . 0}$ & $\mathbf{5 2 5 1 6}$ \\
\cline { 2 - 6 } & Uniman-Prop-Uniman-Vis & 2.3 & 0.8 & 3.8 & 29447 \\
\cline { 2 - 6 } & Uniman-Prop-Biman-NoVis & $\mathbf{- 1 0 . 6}$ & $\mathbf{- 1 2 . 1}$ & $\mathbf{- 9 . 1}$ & $\mathbf{1 7 5 4 6}$ \\
\cline { 2 - 6 } & Uniman-Prop-Biman-Vis & $\mathbf{- 1 1 . 7}$ & $\mathbf{- 1 3 . 3}$ & $\mathbf{- 1 0 . 2}$ & $\mathbf{3 2 5 1 7}$ \\
\hline
\end{tabular}

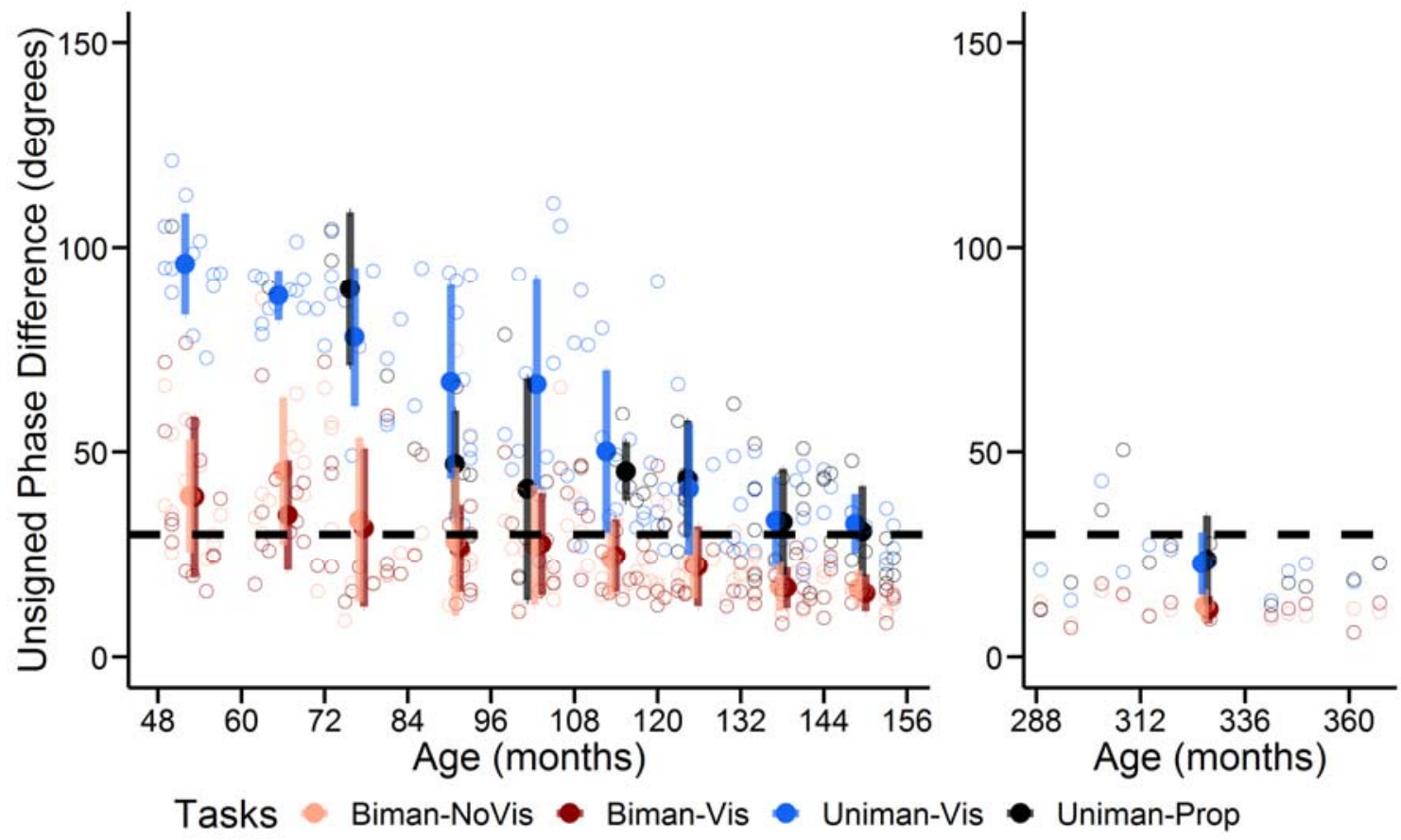

Fig. 2.

Unsigned phase difference between the two hands in bimanual Tasks 1 and 2 (dark and light red) and in unimanual Tasks 3 and $\mathbf{4}$ (blue and black) for children (left panel) and adults (right panel). The dashed lines represent the $30^{\circ}$ threshold under which we considered subjects to perform well. Coloured circles represent the mean for each age group; bars show the s.d. Fewer than half of the 4-8 year-old participants performed Task 4 (Uniman-Prop; black), the means are thus likely to be non-representative (means in Uniman-Prop are not shown for 4- to 5-year-old children, because $\mathrm{n} \leq 2$ ).

\subsubsection{2 | Unsigned phase difference between the hands per age group}

In a second analysis, we grouped children into yearly cohorts and contrasted, for each cohort as well as for an adult group, performance between tasks. Again, we first focus on the bimanual tasks and will return to the unimanual tasks in section 2.2.1.1; Table 2 presents estimates for all four tasks. In brief, performance between the two bimanual tasks did not credibly differ at any age, including adults. There was an exception for the cohort of age 5 (Biman-Vis - Biman-NoVis: $\beta_{\text {Task }}=-11.1, \mathrm{HDI}_{95 \%}$ $=[-15.1 ;-7.2]$ ), for which coordination was worse when no visual feedback was available (compare light and dark red large dots in Figure 2). 
Next, we compared, for each task, performance of each age cohort with that of adults to assess at which age children show adult-like variability in each task. Figure 3 show the estimates for each task and age group (see supplementary Table S1 for detailed statistics).

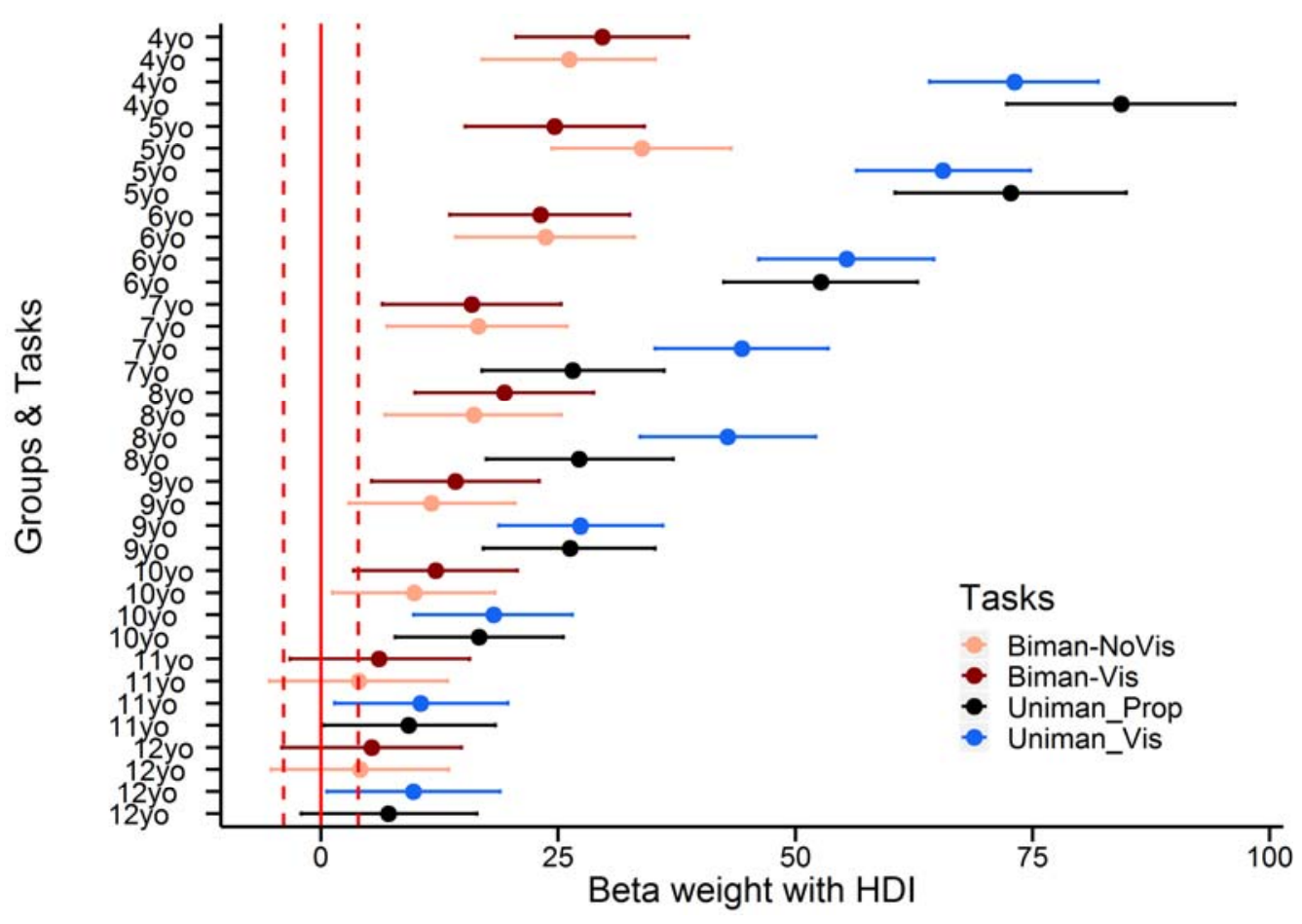

Fig. 3.

Illustration of age effects in Tasks 1-4. The area between whiskers represents the highest density interval (HDI) of a $\beta$ weight's posterior distribution in the model with Age Group as a categorical variable and the Age group adult as reference. Vertical dashed red lines represent the ROPE to establish that an age group differed from adults (ROPE: $[-3.9 ; 3.9])$.

For Biman-NoVis, there was overlap of the HDI and the ROPE for the comparisons of the 9-12 year cohorts with adults, indicating that these age groups did not perform credibly different than adults. The younger cohorts showed higher variability than adults.

For Biman-Vis, the HDI overlapped with the ROPE for the comparison of 10 to 12 year cohorts with adults, but variability was higher for children in the 4-9 year cohorts. In summary, performance in the bimanual task was comparable across almost all age groups, and was indistinguishable from adult performance around age 9-10.

Table 2. Beta Estimates of the within comparison between Tasks $\mathbf{1}$ to $\mathbf{4}$ for each age group. Comparison with Task 4 (Uniman-Prop) was not processed for children aged 4-5 years as only few children per group performed the task $(\leq 2)$. Positive $\beta$ Estimates indicate that performance was worse in the first task of each line. Comparisons in which the difference between tasks is credible are in indicated in bold italic (HDI outside [-3.9; 
3.9]). ESS stands for the Effective Sample Size as calculated during Bayesian model fitting. The Lower and Upper Confidence Interval (Cl) values constitute the 95\% High Density Interval (HDI).

\begin{tabular}{|c|c|c|c|c|c|}
\hline Group & Contrasts & $\begin{array}{c}\boldsymbol{\beta} \\
\text { Estimate }\end{array}$ & $\begin{array}{c}\text { Lower } \\
\mathrm{Cl}\end{array}$ & $\begin{array}{c}\text { Upper } \\
\mathrm{Cl}\end{array}$ & ESS \\
\hline \multirow{6}{*}{ Adults } & Task 2-1: Biman-Vis - Biman-NoVis & -1.8 & -5.1 & 1.4 & 10206 \\
\hline & Task 3-1: Uniman-Vis - Biman-NoVis & 10.1 & 7.0 & 13.2 & 9937 \\
\hline & Task 3-2: Uniman-Vis - Biman-Vis & 11.9 & 8.9 & 15.0 & 22603 \\
\hline & Task 4-3: Uniman-Prop - Uniman-Vis & 0.8 & -2.0 & 3.7 & 27516 \\
\hline & Task 4-1: Uniman-Prop - Biman-NoVis & 10.9 & 7.8 & 14.0 & 9937 \\
\hline & Task 4-2: Uniman-Prop - Biman-Vis & 12.7 & 9.6 & 15.7 & 24608 \\
\hline \multirow{6}{*}{ 12yo } & Task 2 -1: Biman-Vis - Biman-NoVis & -0.6 & -4.1 & 2.8 & 42184 \\
\hline & Task 3-1: Uniman-Vis - Biman-NoVis & 15.6 & 12.5 & 18.9 & 42836 \\
\hline & Task 3-2: Uniman-Vis - Biman-Vis & 16.3 & 13.0 & 19.4 & 55388 \\
\hline & Task 4-3: Uniman-Prop - Uniman-Vis & -1.8 & -4.7 & 1.0 & 50814 \\
\hline & Task 4-1: Uniman-Prop - Biman-NoVis & 13.8 & 10.6 & 17.0 & 42133 \\
\hline & Task 4-2: Uniman-Prop - Biman-Vis & 14.5 & 11.4 & 17.7 & 53941 \\
\hline \multirow{6}{*}{ 11yo } & Task 2 - 1: Biman-Vis - Biman-NoVis & 0.3 & -3.0 & 3.5 & 47841 \\
\hline & Task 3-1: Uniman-Vis - Biman-NoVis & 16.6 & 13.5 & 19.7 & 44550 \\
\hline & Task 3-2: Uniman-Vis - Biman-Vis & 16.3 & 13.2 & 19.4 & 54139 \\
\hline & Task 4-3: Uniman-Prop - Uniman-Vis & -0.4 & -3.2 & 2.5 & 52734 \\
\hline & Task 4 - 1: Uniman-Prop - Biman-NoVis & 16.2 & 13.1 & 19.3 & 44548 \\
\hline & Task 4-2: Uniman-Prop - Biman-Vis & 15.9 & 12.8 & 18.9 & 52734 \\
\hline \multirow{6}{*}{ 10yo } & Task 2 -1: Biman-Vis - Biman-NoVis & 0.4 & -2.3 & 3.1 & 45014 \\
\hline & Task 3-1: Uniman-Vis - Biman-NoVis & 18.4 & 15.9 & 20.9 & 46753 \\
\hline & Task 3-2: Uniman-Vis - Biman-Vis & 18.0 & 15.5 & 20.5 & 51275 \\
\hline & Task 4 - 3: Uniman-Prop - Uniman-Vis & -0.7 & -4.2 & 2.8 & 49678 \\
\hline & Task 4-1: Uniman-Prop - Biman-NoVis & 17.7 & 14.0 & 21.3 & 45732 \\
\hline & Task 4-2: Uniman-Prop-Biman-Vis & 17.3 & 13.6 & 20.9 & 49678 \\
\hline \multirow{6}{*}{ 9yo } & Task $2-1$ : Biman-Vis - Biman-NoVis & 0.7 & -2.3 & 3.6 & 45100 \\
\hline & Task 3-1: Uniman-Vis - Biman-NoVis & 25.7 & 23.0 & 28.5 & 44843 \\
\hline & Task 3-2: Uniman-Vis - Biman-Vis & 25.1 & 22.5 & 27.9 & 51449 \\
\hline & Task 4-3: Uniman-Prop - Uniman-Vis & -0.2 & -3.5 & 3.2 & 51457 \\
\hline & Task 4 - 1: Uniman-Prop - Biman-NoVis & 25.5 & 21.8 & 29.1 & 44764 \\
\hline & Task 4-2: Uniman-Prop-Biman-Vis & 24.8 & 21.2 & 28.2 & 53298 \\
\hline \multirow{6}{*}{8 yo } & Task 2-1: Biman-Vis - Biman-NoVis & 1.3 & -2.1 & 4.6 & 44391 \\
\hline & Task 3-1: Uniman-Vis - Biman-NoVis & 36.8 & 33.6 & 40.1 & 44130 \\
\hline & Task 3-2: Uniman-Vis - Biman-Vis & 35.5 & 32.3 & 38.6 & 52690 \\
\hline & Task 4 - 3: Uniman-Prop - Uniman-Vis & -14.8 & -19.3 & -10.5 & 50880 \\
\hline & Task 4-1: Uniman-Prop - Biman-NoVis & 22.0 & 17.4 & 26.6 & 43045 \\
\hline & Task 4-2: Uniman-Prop - Biman-Vis & 20.6 & 16.1 & 25.2 & 50880 \\
\hline \multirow{6}{*}{ 7yo } & Task 2-1: Biman-Vis - Biman-NoVis & -2.6 & -6.1 & 0.7 & 43507 \\
\hline & Task 3-1: Uniman-Vis - Biman-NoVis & 37.9 & 34.7 & 41.1 & 44120 \\
\hline & Task 3-2: Uniman-Vis - Biman-Vis & 40.5 & 37.4 & 43.6 & 54290 \\
\hline & Task 4-3: Uniman-Prop - Uniman-Vis & -17.0 & -21.1 & -13.1 & 53270 \\
\hline & Task 4-1: Uniman-Prop - Biman-NoVis & 20.9 & 16.6 & 25.0 & 43510 \\
\hline & Task 4-2: Uniman-Prop - Biman-Vis & 23.4 & 19.4 & 27.7 & 55335 \\
\hline 6yo & Task 2 - 1: Biman-Vis - Biman-NoVis & -2.4 & -5.7 & 1.2 & 44214 \\
\hline
\end{tabular}




\begin{tabular}{|c|c|c|c|c|c|}
\hline & Task 3-1: Uniman-Vis - Biman-NoVis & 41.8 & 38.6 & 45.0 & 45359 \\
\hline & Task 3-2: Uniman-Vis-Biman-Vis & 44.2 & 40.9 & 47.3 & 54585 \\
\hline & Task 4 -3: Uniman-Prop - Uniman-Vis & -1.8 & -6.7 & 3.0 & 48897 \\
\hline & Task 4-1: Uniman-Prop - Biman-NoVis & 39.9 & 34.9 & 44.9 & 43111 \\
\hline & Task 4 - 2: Uniman-Prop - Biman-Vis & 42.3 & 37.3 & 47.4 & 49675 \\
\hline \multirow{3}{*}{5 yo } & Task 2-1: Biman-Vis-Biman-NoVis & -11.1 & -15.1 & -7.2 & 40397 \\
\hline & Task 3-1: Uniman-Vis - Biman-NoVis & 41.7 & 38.3 & 45.1 & 43051 \\
\hline & Task 3-2: Uniman-Vis - Biman-Vis & 52.8 & 49.3 & 56.2 & 52421 \\
\hline \multirow{3}{*}{4 yo } & Task 2-1: Biman-Vis - Biman-NoVis & 1.6 & -1.9 & 5.1 & 43354 \\
\hline & Task 3-1: Uniman-Vis-Biman-NoVis & 56.9 & 53.7 & 60.1 & 43111 \\
\hline & Task 3-2: Uniman-Vis - Biman-Vis & 55.4 & 52.3 & 58.3 & 53084 \\
\hline
\end{tabular}

\subsubsection{Signed phase difference between the two hands}

Analysis up to now has focused on variability in coordinating the two hands. Next, we asked whether one hand had a constant offset relative to the other, that is, whether one hand regularly leads the other by a few degrees. We ran the same Bayesian model as the one used for variability, but with the signed error as dependent measure. Figure 4 shows that the signed error was generally negative for both unimodal tasks, without a clear change across ages. In the model, the intercept differed from zero for both tasks (Biman-NoVis: $\beta_{\text {Intercept }}=-15.6, \mathrm{HDI}_{95 \%}=[-24.8 ;-6.2]$; Biman-Vis: $\beta_{\text {Intercept }}=-17.3$, $\mathrm{HDI}_{95 \%}=[-26.4 ;-8.1]$ ), but neither the continuous Age predictor (Biman-NoVis: $\beta_{\text {Age }}=2.7, \mathrm{HDI}_{95 \%}=[-$ 0.0; 5.2]; Biman-Vis: $\left.\beta_{\text {Age }}=3.1, \mathrm{HDl}_{95 \%}=[0.6 ; 5.7]\right)$, nor the Task $\left(\beta=-1.8, \mathrm{HDI}_{95 \%}=[-8.2 ; 4.7]\right)$ or the Task*Age interaction $\left(\beta=0.6, \mathrm{HDI}_{95 \%}=[-1.2 ; 2.4]\right)$ contrasts between the two bimanual tasks were credibly different from 0 . Together, these results indicate that the dominant hand lagged behind the non-dominant hand for all ages.

\section{2 | Unimanual motor coordination}

\subsubsection{Unsigned phase difference between hand and external signal}

\subsubsection{Unsigned phase difference between hand and external signal across all ages}

We now turn to the analysis of the unimanual coordination tasks. In these tasks participants had to synchronize the movement of their dominant hand with a circling dot (Uniman-Vis) or with the passively moved, non-dominant hand (Uniman-Prop). Figure 2 shows that, as in the bimanual tasks, in unimanual tasks coordination improved with age (blue and black dots). The respective slope coefficients were negative and different from 0 (Uniman-Vis: $\beta_{\text {Age }}=-21.8, \mathrm{HDI}_{95 \%}=[-23.7 ;-19.7]$; Uniman-Prop: $\left.\beta_{\text {Age }}=-19.5, \mathrm{HDl}_{95 \%}=[-21.8 ;-17.1]\right)$, statistically confirming the effect of age. There was a credible difference for the contrast between the two tasks, indicating that the Intercept coefficient was smaller for Uniman-Prop than Uniman-Vis $\left(\beta=-12.1, \mathrm{HDI}_{95 \%}=[-17.9 ;-5.8]\right)$. However, there was 
no credible Task*Age interaction contrast (see Table 1), indicating that the rate of performance improvement did not differ between unimanual tasks. However, both unimanual tasks exhibited large effects of Task and Task*Age interactions when contrasted with the bimanual tasks (see Table 1 for all model estimates). Unimanual intercepts were higher, and the negative slopes were steeper, indicating that the performance was worst at younger ages, and the improvement with age larger, in the unimanual than in the bimanual tasks.

\subsubsection{Unsigned phase difference between hand and external signal per age group}

Analysis by yearly cohorts confirmed this overall result pattern across all experimental groups, including adults (see Table 2). Thus, coordination was better for bimanual than for unimanual tasks, although this difference was more pronounced for younger than for older children.

Finally, we assessed at what age unimanual performance of children likened that of adults (see Table S1 in the supplementary information). There was no credible difference in performance in UnimanVis and Uniman-Prop for 11 and 12 year-old children as compared to adults, whereas all other age groups performed worse than adults. As for Biman-NoVis and Biman-Vis, performance in Uniman-Vis and Uniman-Prop gradually improved over age (see Fig. 3), and we did not observe a step at any point in the developmental trajectory.

\subsubsection{Signed phase difference between hand and external signal}

\subsubsection{I Signed phase difference between hand and external signal across all ages}

If a participant moves the hand following an external guiding signal, there should be a constant offset between the guiding signal and the hand, given that processing of sensory information takes time. If, in contrast, the hand maintained a zero phase lag, this would imply that the hand is moved predictively. Figure 4 shows that the signed error for unimanual tasks resembled that for bimanual tasks in that it was generally negative and without a clear change across ages. However, model analysis indicated that the Task intercept for Uniman-Prop was different from zero and also credibly more negative than Uniman-Vis (Uniman-Vis: $\beta=-2.0, \mathrm{HDl}_{95 \%}=[-10.7 ; 6.8]$; Uniman-Prop: $\beta=-19.6$, $\mathrm{HDl}_{95 \%}=[-30.8 ;-7.7]$; Task contrast: $\left.\beta=-17.5, \mathrm{HDl}_{95 \%}=[-26.5 ;-8.3]\right)$. Neither Age (Uniman-Vis: $\beta_{\text {Age }}=-$ 3.5, $\mathrm{HDI}_{95 \%}=[-5.9 ;-1.0]$; Uniman-Prop: $\left.\beta_{\text {Age }}=1.7, \mathrm{HDI}_{95 \%}=[-1.3 ; 4.8]\right)$ nor the Task*Age interaction $\left(\beta_{\text {Interaction }}=5.2, \mathrm{HDl}_{95 \%}=[2.9 ; 7.5]\right)$ contrasts between the two unimanual tasks were credibly different from 0 , implying similar performance across ages. Together, these results indicate that at all ages the hand moved predictively in the visual task but followed the external guiding signal in the proprioceptive task. 


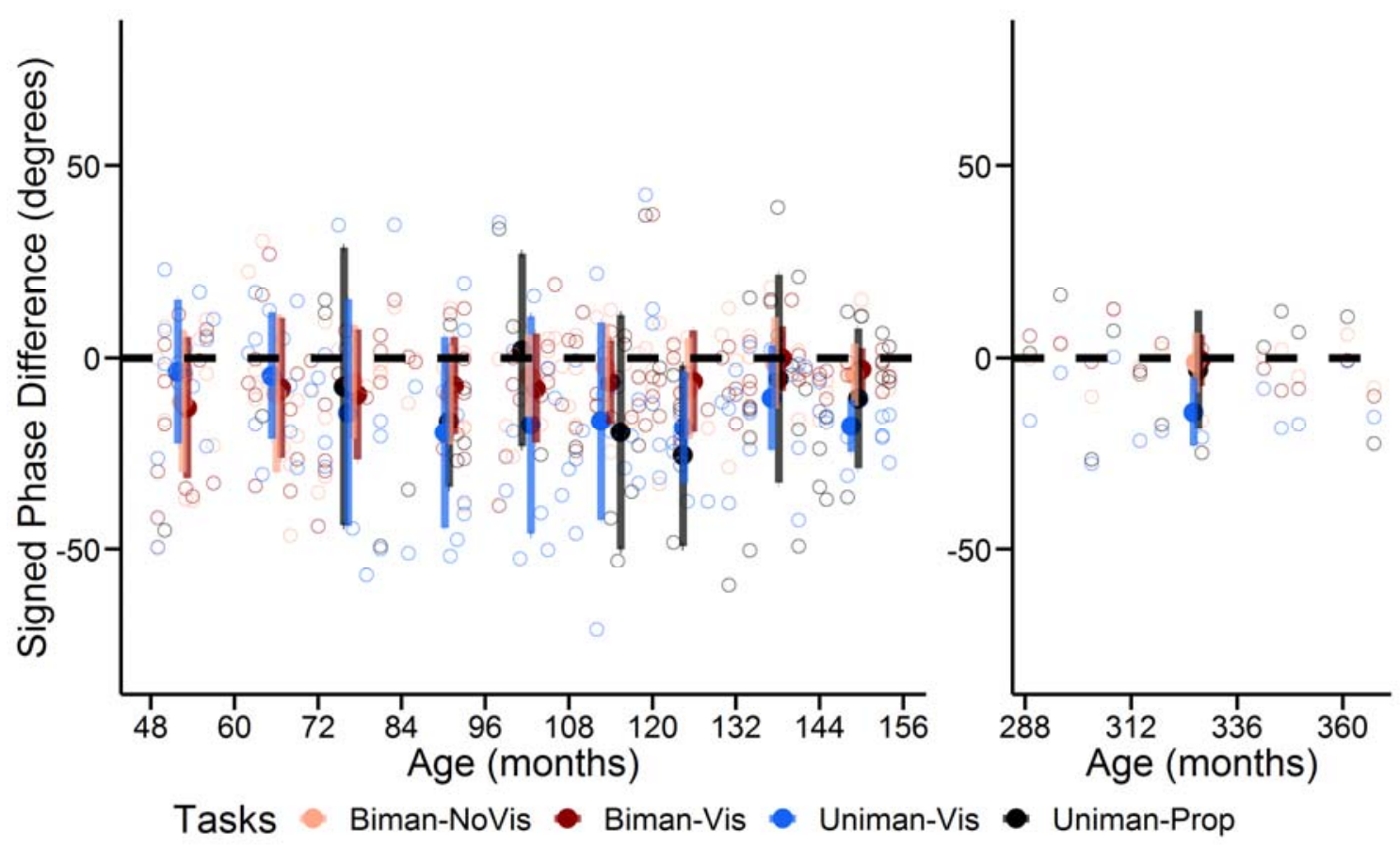

Fig. 4.

Signed phase difference between the two hands in bimanual Tasks 1 and 2 (dark and light red) and in unimanual Tasks 3 and 4 (blue and black) for children (left panel) and adults (right panel). Coloured circles represent the mean for each age group; bars show the s.d. Fewer than half of the 4-8 year-old participants performed Task 4 (Uniman-Prop; black), the means are thus likely to be non-representative (means in UnimanProp are not shown because $n \leq 2$ )

\subsubsection{I Signed phase difference between hand and external signal per age group}

Although the intercept of Uniman-Vis did not differ from 0 overall in the previous analysis, visual inspection suggested that this conclusion may be overly conservative. Therefore, we tested the difference of the intercept against zero separately for each age group. Beta estimates are listed in Table S3 in the supplementary information. Adults lagged in Uniman-Vis $\left(\beta=-14.2, \mathrm{HDI}_{95 \%}=[-21.9 ;-\right.$ 6.5], but not in Uniman-Prop ( $\left.\beta=-3.2, \mathrm{HDI}_{95 \%}=[-11.1 ; 4.4]\right)$. Most children cohorts exhibited lag in Uniman-Vis. For Uniman-Prop. However, participant numbers were low up to age 8, so we refrain from reporting their statistics. Lag was evident for children at ages 9-10, but it decreased thereafter. Performance was indistinguishable between the 12-year-olds and adults. Thus, the cohort-wise analysis rendered qualitatively different results than the overall analysis; given these differences between analyses, their interpretation should be tentative. 


\subsection{Sensory dominance in bimanual motor coordination}

\subsubsection{Unsigned phase difference between transformed hand positions}

\subsubsection{Unsigned phase difference between transformed hand positions across all ages}

As a third aspect of motor control we investigated the ability of children to perform asymmetric coordination, with veridical feedback of hand position (Task 5 - 2:3-NoTransform), or transformed feedback (Task 6 - 2:3-Transform). We then tested the learning of asymmetric coordination (Task 7 2:3-Retention). We analyzed Tasks 5 to 7 separately from Tasks 1-4, because they required different speeds of the two hands, and, thus, the dependent measures were not identical between symmetrical and asymmetrical tasks. We report on the 2:3-Retention (Task 7) in the Supplementary Information, because none of our participants, including adults, performed better than chance in this task. The model was equivalent to that for Tasks 1-4. Because most of the youngest participants did not perform the 2:3 tasks, the standardized unit of age differed from the previous analyses and was 24.6 months.

Figure 5 shows that performance in 2:3-Transform improved with age (green dots), as shown by the negative, non-zero slope coefficient $\left(\beta_{\text {Age }}=-13.5, H_{1} I_{95 \%}=[-15.6 ;-11.3]\right)$. The negative slope value indicates that the variability of the phase difference between hands decreased by the respective amount of degrees with each standardized age unit of 24.6 months. In contrast, performance in the 2:3-NoTransform did not credibly vary with age (Fig. 5, yellow dots; $\beta_{\text {Age }}=-4.2, \mathrm{HDl}_{95 \%}=[-6.40 ;-2.0]$ ). There was a credible difference between tasks for both Task and Task*Age interaction contrasts, indicating that the intercept and rate of improvement was smaller for 2:3-NoTransform than 2:3Transform $\left(\beta_{\text {Task }}=28.6, \mathrm{HDl}_{95 \%}=[22.7 ; 34.5] ; \beta_{\text {Interaction }}=-9.3, \mathrm{HDl}_{95 \%}=[-10.6 ;-8.1]\right)$. During a $2: 3$ coordination, the correct phase difference between the two hands changes from moment to moment. If a participant incorrectly performed symmetrical (i.e., 1:1) coordination, a pattern that often emerges automatically, then the resulting variable error will be about $83^{\circ}$. In Fig. 5, we plot this error as reference, together with participants' performance. Note, that performance in 2:3NoTransform (Fig. 5, yellow color) mostly scatters around the $83^{\circ}$ line across all ages, including adults. Thus, participants were unsuccessful with veridical feedback across all age groups. In contrast, performance improved in the older groups (Fig. 5, green color). 


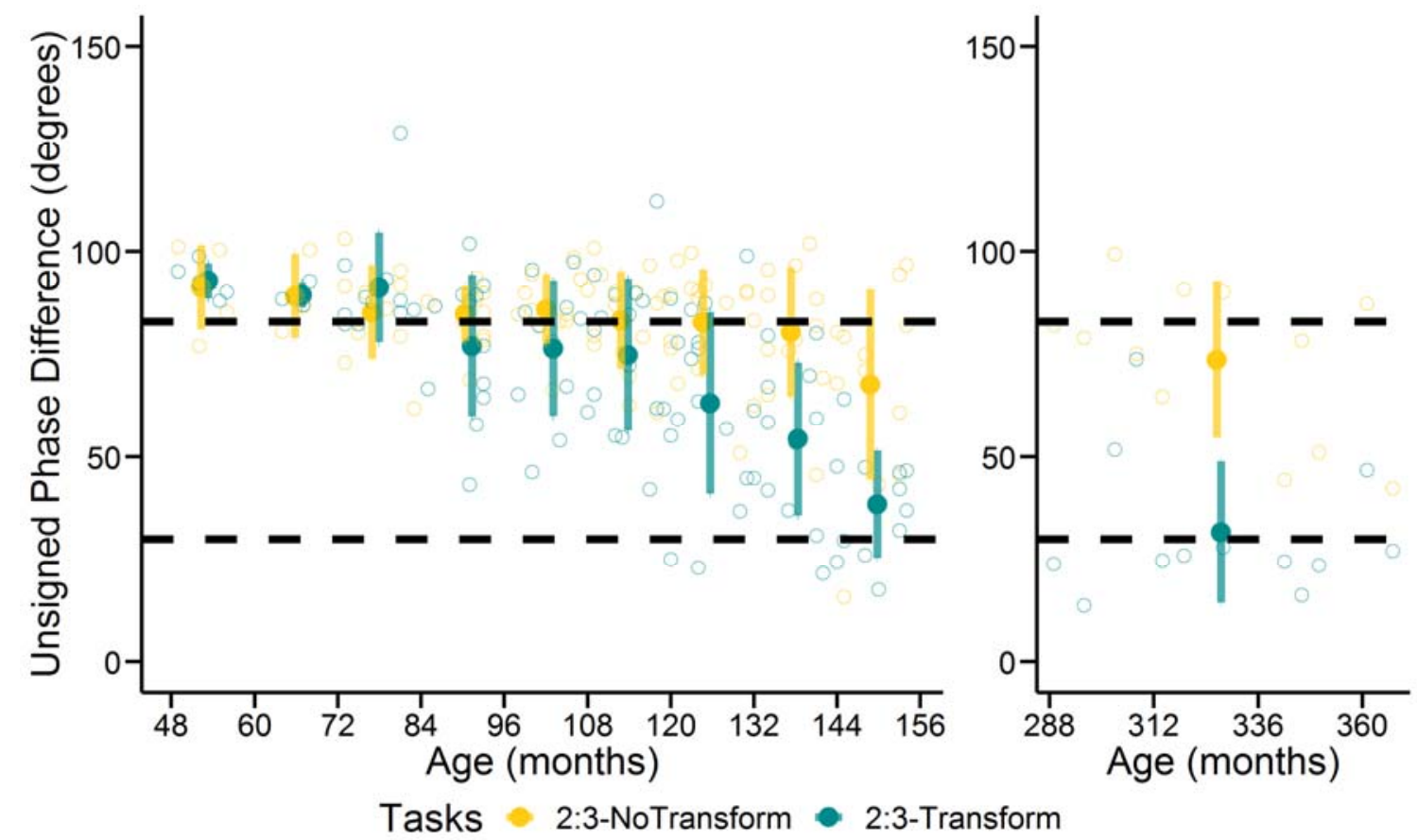

Fig. 5.

Unsigned phase difference between the two hands in the 2:3 coordination tasks for children (left panel) and adults (right panel). The lowest dashed lines represent the $30^{\circ}$ threshold under which we considered subjects to perform well. The higher dashed line $\left(83^{\circ}\right)$ represents the phase angle when participants (incorrectly) perform 1:1 coordination. Coloured circles are the mean for each age group; error bars denote s.d. Fewer than half of the 4-5 year-old participants performed Task 5 (yellow), the means are thus likely to be nonrepresentative.

\subsubsection{Unsigned phase difference between transformed hand positions per age group}

Next, we contrasted performance between 2:3-NoTransform and 2:3-Transform within each age group. Beta estimates for these comparisons are shown in Table 3. In brief, performance between the two tasks credibly differed in all age groups older than 9, suggesting a consistent use of transformed visual feedback to perform the coordination for these participants. A credible difference was also present at age 7 , but it was absent at 8 years. Children younger than 7 were unable to use transformed feedback (Table 3).

Table 3. Beta Estimates of the within comparison between Tasks 5 and 6 for each age group. Negative $\beta$ Estimates indicate that performance was better in Task 6 (2:3-Transform) than Task 5 (2:3-NoTransform). Credible difference between the tasks in indicated in bold italic (HDI outside [-5.4; 5.4]). ESS stands for the Effective Sample Size as calculated by the Bayesian model. The Lower and Upper Confidence Interval values constitute the 95\% High Density Interval (HDI).

\begin{tabular}{|c|c|c|c|c|}
\hline Groups & $\beta$ Estimate & Lower Cl & Upper Cl & ESS \\
\hline Adults & -45.0 & -48.8 & -41.1 & 4152 \\
\hline
\end{tabular}




\begin{tabular}{|c|c|c|c|c|}
\hline 12 yo & -29.6 & -33.7 & -25.5 & 43277 \\
\hline 11 yo & -28.3 & -31.8 & -24.7 & 43270 \\
\hline 10 yo & -18.4 & -21.5 & -15.6 & 53326 \\
\hline 9 yo & -8.6 & -11.6 & -5.6 & 50097 \\
\hline 8 yo & -8.7 & -12.4 & -4.7 & 45141 \\
\hline 7 yo & -10.3 & -14.3 & -6.4 & 48786 \\
\hline 6 yo & 1.6 & -2.3 & 5.5 & 45047 \\
\hline 5 yo & -0.8 & -9.0 & 7.3 & 33838 \\
\hline 4 yo & 1.6 & -5.0 & 8.2 & 31765 \\
\hline
\end{tabular}

Comparison of each age group's performance in 2:3-NoTransform with that of adults did not reveal a credible difference for any age cohort, in line with the above result that 2:3-NoTransform did not improve with age (see Fig. 6; statistics reported in Table S2 of the supplementary information). In the 2:3-Transform, the adult group performed better than all age cohorts except the 12 year-olds (see Fig. $\left.6 ; \beta=10.1, \mathrm{HDI}_{95 \%}=[0.5 ; 19.8]\right)$.

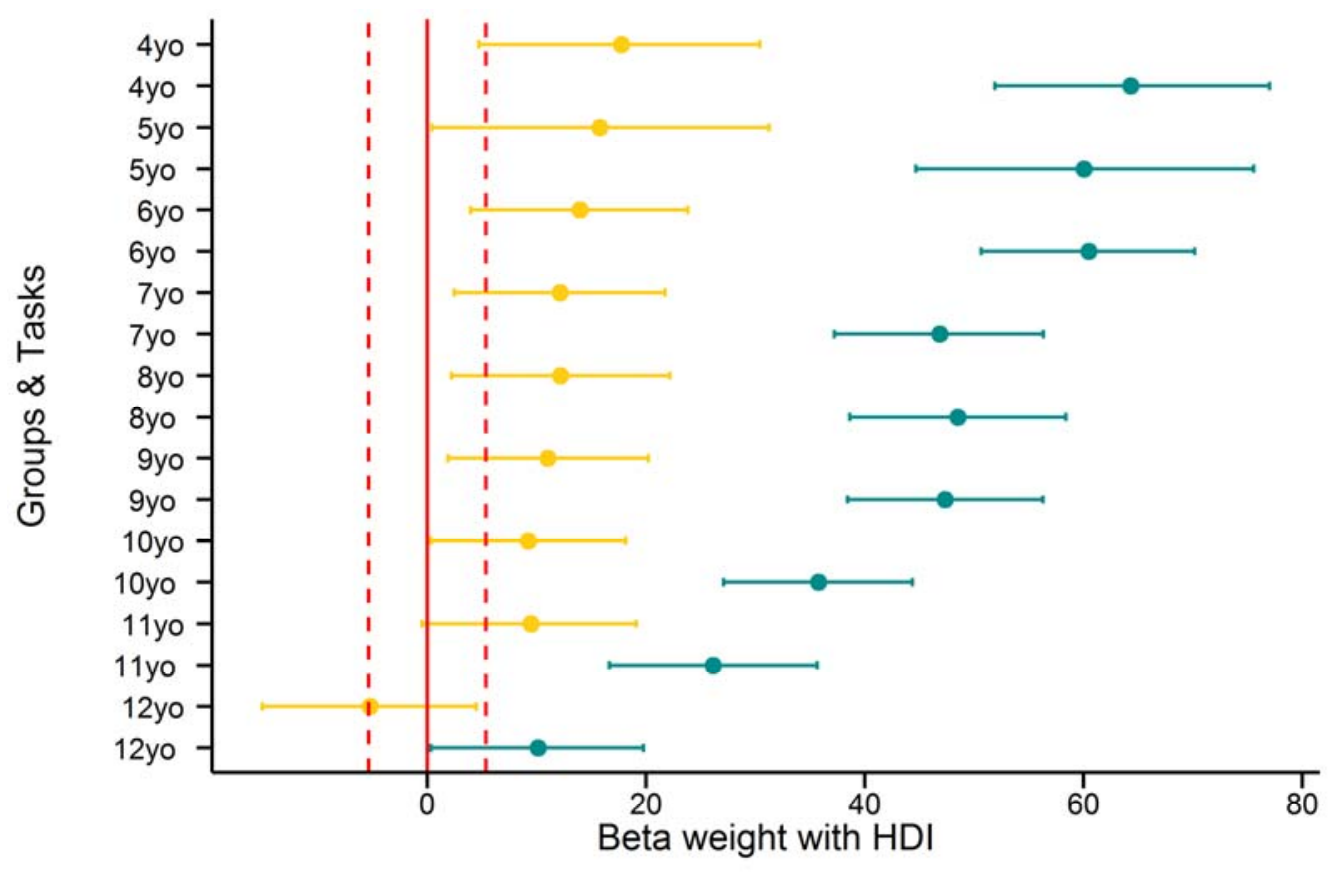

Tasks 2:3-NoTransform $\bullet$ 2:3-Transform

Fig. 6.

Illustration of age effects in Tasks 5 (2:3-NoTransform) and 6 (2:3-Transform). The area between whiskers represents the highest density interval (HDI) of a $\beta$ weight's posterior distribution obtained with Age Group as a categorical variable and the Age group adult as reference. Vertical dashed red lines represent the ROPE to establish that an age group differed from adults (ROPE: [-5.4; 5.4]). 
Note, that we did not assess the signed difference for the 2:3 coordination, because the instructions were to be faster with one hand, and whether one or the other transformed cursor leads the other would not be informative about general aspects of coordination.

\section{4 | Relationship between tasks beyond improvement with age}

\subsection{1 | Relationship between bimanual tasks}

Our analysis has so far shown that both Biman-NoVis and Biman-Vis improve with age, and that, overall, this development is similar for the two tasks. However, this conclusion pertains to the average performance of all children. In other words, it is possible that performance globally improves with age, implying that the seemingly similar average development is trivially explained by unspecific factors. To assess whether performance in the two tasks is related beyond a global age improvement, we computed their correlation with age partialed out. The partial correlation was $0.70\left(\mathrm{HDI}_{95 \%}=\right.$ [0.57; 0.84]; $\mathrm{n}=120$; two-sided test; $\mathrm{BF}=2.60 \mathrm{e} 15 ; \mathrm{BF}$ expresses the odds of an $\mathrm{H} 1$ of true correlation compared to an $\mathrm{HO}$ of zero correlation). This high correlation implies that a child that was good in one task was also good in the other, and, thus, suggests that the two tasks are driven by common underlying development.

\subsubsection{Relationship between bimanual and unimanual tasks}

Next, we compared within-participant performance across tasks through correlations between all pairs of tasks, with age partialed out (see Table 4). Like the two bimanual tasks, the two unimanual tasks exhibited high correlation $\left(\rho=0.6 ; \mathrm{HDI}_{95 \%}=[0.3 ; 0.85]\right)$, though the confidence interval was wide due to the low $\mathrm{N}$ available for Uniman-Prop. In contrast, correlations between uni- and bimanual tasks were smaller, ranging from .28 to .42 , with only the higher correlations exhibiting BFs that suggest reliable relationships. Counterintuitively, higher correlations were obtained for BimanNoVis with Uniman-Vis and for Biman-Vis with Uniman-Prop. For the two visual uni- and bimanual tasks (Biman-Vis and Uniman-Vis) and the two proprioceptive tasks (Biman-NoVis and Uniman-Prop), correlations were estimated to be lower; however, the wide HDIs again indicate that $\mathrm{N}$ is too low to draw firm conclusions about comparisons including Uniman-Prop.

Note, for Uniman-Prop, participants had to let their non-dominant hand be passively guided. It is theoretically possible that children did not conform with this instruction. If children had, instead, actively moved their non-dominant hand, then they would effectively have performed a bimanual coordination task without vision, i.e., Biman-NoVis. Both the much worse performance of the unimanual tasks, and the lower correlations between unimanual and bimanual tasks than those 
between the bimanual tasks, indicate that children did not perform bimanual coordination and, thus, did not actively synchronize their hands, during Uniman-Prop.

\subsection{3 | Relationship between asymmetric coordination tasks}

Finally, we asked how performance in the 2:3 tasks was related amongst each other and with the 4 symmetrical tasks within participants (see Table 4). Partial correlation between the two 2:3 tasks, partialing out age, revealed a credible correlation of $0.40\left(\mathrm{HDI}_{95 \%}=[0.17 ; 0.64]\right.$; two-sided test; $\mathrm{n}=$ $100, B F=23.2$ ). Thus, a child who performed well with veridical feedback performed well also with transformed feedback, and a child who performed badly in the veridical feedback task gained less from transformed feedback. Correlations with the symmetrical tasks were overall low. They were lowest with the bimanual tasks, and higher, though with moderate reliability, for the uni-manual tasks.

Table 4. Two-sided correlations between Tasks 1 to 6, with Age partialed out. Credible correlations are in italic bold. Lower and Upper Confidence Interval values constitute the 95\% HDI.

\begin{tabular}{|l|c|c|c|c|c|}
\hline \multicolumn{1}{|c|}{ Tasks } & Coefficient & Lower Cl & Upper Cl & BF & Sample (N) \\
\hline Biman-NoVis / Biman-Vis & $\mathbf{0 . 7 0}$ & $\mathbf{0 . 5 7}$ & $\mathbf{0 . 8 4}$ & $\mathbf{2 . 6 0 e 1 5}$ & $\mathbf{1 2 0}$ \\
\hline Biman-NoVis / Uniman-Vis & $\mathbf{0 . 4 2}$ & $\mathbf{0 . 1 8}$ & $\mathbf{0 . 6 7}$ & $\mathbf{1 6 . 5}$ & $\mathbf{1 1 9}$ \\
\hline Biman-NoVis / Uniman-Prop & 0.29 & -0.06 & 0.64 & 0.44 & 51 \\
\cline { 2 - 6 } Biman-Vis / Uniman-Vis & 0.28 & 0.02 & 0.54 & 0.60 & 119 \\
\hline Biman-Vis / Uniman-Prop & $\mathbf{0 . 4 0}$ & $\mathbf{0 . 1 0}$ & $\mathbf{0 . 6 9}$ & $\mathbf{3 . 4}$ & $\mathbf{5 1}$ \\
\hline Uniman-Vis / Uniman-Prop & $\mathbf{0 . 5 8}$ & $\mathbf{0 . 3 0}$ & $\mathbf{0 . 8 5}$ & $\mathbf{2 6 4}$ & $\mathbf{5 1}$ \\
\cline { 2 - 6 } 2:3-NoTransform / 2:3-Transform & $\mathbf{0 . 4 0}$ & $\mathbf{0 . 1 7}$ & $\mathbf{0 . 6 4}$ & $\mathbf{2 3 . 2}$ & $\mathbf{1 0 0}$ \\
\hline Biman-NoVis / 2:3-Transform & 0.08 & -0.09 & 0.26 & 0.17 & 101 \\
\hline Biman-Vis / 2:3-Transform & 0.10 & 0.07 & 0.27 & 0.22 & 101 \\
\hline Uniman-Vis / 2:3-Transform & $\mathbf{0 . 3 0}$ & $\mathbf{0 . 0 8}$ & $\mathbf{0 . 5 2}$ & $\mathbf{3 . 0}$ & $\mathbf{1 0 0}$ \\
\hline Uniman-Prop / 2:3-Transform & 0.33 & 0.04 & 0.62 & 1.49 & 50 \\
\hline
\end{tabular}

\section{3 | DISCUSSION}

This study investigated three aspects of motor control in children aged 4-12. First, symmetrical bimanual tasks assessed the ability of children to coordinate motor commands, vision, and proprioception for symmetrical bimanual coordination. Children performed these tasks well already at age 4. However, performance exhibited comparable improvement with age for both sensory contexts. Second, symmetrical unimanual tasks assessed coordination with a sensory (visual or passive-proprioceptive) signal. Young children performed much worse in these tasks, and performance improved dramatically with age. Third, 2:3 tasks assessed the development of the ability to separate different sensory modalities and focus on transformed symmetrical visual feedback to perform asymmetrical movements. While all participants, including adults, were unable 
to perform asymmetric coordination, providing transformed visual feedback improved the coordination in some individuals already at age 7 , though more regularly starting at age 9 .

Our study revealed that children successfully performed bimanual coordination already at age 4. Yet, variability decreased from ages 4 to 12 , indicating refinement of the performance and increased accuracy. Adding visual feedback during symmetric bimanual coordination did not improve coordination. Thus, although other paradigms have revealed that bimanual coordination can rely on visual information (Brandes et al., 2017; Mechsner et al., 2001), our result suggests that coordination in standard symmetrical tasks proceeds independent of visual guidance. This conclusion implies that either bimanual coordination relies exclusively on somatosensory (proprioceptive) information to perform circling movements, or that it relies predominately on coordinated motor signals sent through the corpus callosum. This latter interpretation is supported by studies on neurological patients: bimanual performance was preserved in absence of proprioception, whether vision was available or not (Spencer et al., 2005); in contrast, callosotomy patients were unable to maintain symmetric coordination (Kennerley, Diedrichsen, Hazeltine, Semjen, \& Ivry, 2002). Thus, symmetrical bimanual coordination may generally depend on motor connectivity more than on sensory information.

When children had to coordinate with a sensory signal in the unimanual symmetric tasks, the unsigned phase difference was comparable for both visual and proprioceptive sensory input. Moreover, performance in the two tasks was correlated. Together, these independent findings suggest a common developmental process for the use of sensory input during motor coordination. Furthermore, unsigned phase difference was severely impaired in the younger groups, with a real improvement occurring only for the oldest groups. The fact that development of unimanual coordination lags several years behind that of bimanual coordination calls into question the idea that bimanual coordination is merely a special case of coordination in general (Bingham, 2004). Instead, our present results rather suggest that (symmetrical) bimanual coordination is a qualitatively different capability of the motor system than other types of coordination, due to the ability to use coordinated motor commands instead of having to wait for sensory feedback.

Despite the commonalities between the visual and the proprioceptive task, there was a credible difference between the two tasks in the signed phase difference for the oldest children; a non-zero value of this measure implies a constant phase error between the guiding signal and the active hand. Just like adults, older children produced feedback-based, lagging movement when following a visual cursor, but moved predictively when following their own, passively moved hand. 
The difficulties of the younger participants and the late emergence of predictive movement in unimanual coordination strike as surprising, given that children can integrate visual and proprioceptive feedback to correct their movement online already at age 4 (e.g. Contreras-Vidal, Bo, Boudreau, \& Clark, 2005; Konczak, Jansen-Osmann, \& Kalveram, 2003). This divergence in skill may depend on task differences: motor adaptation studies apply forces or visuomotor rotations directly to (feedback about) the moving hand during short, discrete movements. In our study, children had to use, in addition, information about an external, moving cue, which may require different cognitive resources than responding to limb perturbation. Furthermore, coordination during continuous movement, as used here, differs from coordination in short, goal-directed movements: in experiments in which goal-directed movements are performed repeatedly, each new movement can be adapted according to the registered error of the previous movement(s). In contrast, continuous coordination requires integrating the external cue to derive the necessary correction of the ongoing movement. These task features appear to rely on mechanisms that develop later than those responsible for online corrections of discrete movements.

Adding visual transformed feedback in the 2:3 coordination task consistently improved coordination in children from 9 to 12 years of age. Performance in this task moderately correlated with the ability of each child to integrate visual feedback independently of age. This finding suggests that the ability to integrate visual feedback was an important factor for task success, consistent with the task requirements. The ability to perform the task was evident around age 9 , in line with a previous study on the use of Lissajous displays by children (Leinen et al., 2016). This is also the age at which children first exhibit adult-like optimal multisensory integration (Burr \& Gori, 2012; Gori, 2015). Thus, the ability to strategically ignore, or down-weight, proprioception and conflicting motor commands in response to task demands appears to require mature multisensory integration abilities.

It is notew orthy that some children were able to use visual feedback to improve coordination as early as age 7, but 8-year-old children did not show this ability. Although this conclusion is based on a statistical between-group comparison, this result fits with the many previous reports of performance decrements around the age of 8 years (Badan, Hauert, \& Mounoud, 2000; Hay, 1978; Pellizzer \& Hauert, 1996; Smyth, Katamba, \& Peacock, 2004). Under this notion, adult-like motor control strategies are implemented at age 8 , which leads to transient disorganization of the motor processes that were at work until this point.

To summarize, the performance observed in seven related tasks by large sample of 4-12 year-old children suggests interdependent, serial development of different motor control functions. Mastering of simple, symmetrical bimanual coordination may rely predominately on motor-related interhemispheric transfer and develop first. Yet, even this seemingly basic ability matures until about 
age 9. Unimanual synchronization with externally produced signals develops later, and proceeds until about age 11. As there is no motor command from the non-dominant hand, accurate coordination requires the integration of sensory feedback and online control of the active hand; accordingly, this task cannot be solved by relying on motor commands alone. Nonetheless, this ability depends, in part, on bimanual ability, as suggested by the moderate correlations between bimanual and unimanual tasks in our sample. What could be the common mechanism shared by the two forms of coordination? Both tasks rely on comparing two ongoing signals: the two motor commands in symmetric bimanual coordination, and a motor command and a sensory channel in unimanual coordination. In current models of motor control, such comparison is denoted as state estimation, and our experimental result suggests that it is this function that tunes the development of sensorimotor performance (King, Oliveira, Contreras-Vidal, \& Clark, 2012). Finally, selecting rather than integrating multisensory information matured even later, first being adult-like at age 12. Again, a moderate correlation with unimanual task performance suggests the dependence of this function on those motor functions that develop earlier. Notably, the lack of a correlation of 2:3 coordination with bimanual performance implies that the correlations of these two tasks with the unimanual tasks are based on different variance portions of the unimanual task. This finding makes intuitive sense given that the 2:3 coordination requires the exact opposite of symmetrical bimanual coordination, namely to dissociate, rather than synchronize, movement of the two hands. In conclusion, our study challenges the notion that bimanual coordination is just a sub-category of general motor coordination, and instead highlights a complex interdependence of the development of different motor functions.

\section{4 | MATERIALS AND METHODS}

\subsection{Participants and ethical approval}

We enrolled children who signed up at diverse public science popularization events, school visits to our lab, or through flyers, until we had recruited at least 12 children of each age from 4-12 years that met our inclusion criteria. Of 138 tested children, 120 were included in the final sample (52 girls, 68 boys). Additionally, we enrolled a control group of 12 adults ( 6 female, mean age: 27.2 years; s.d.: 2.2 years). All included participants had normal or corrected-to-normal vision and were free of any known neurological, visual and tactile impairments and disorders. Furthermore, children had to have been born in regular term ( $>37^{\text {th }}$ gestational week), score between 70 and 130 on a short IQ test, and pass screening for attentional disorders, learning disabilities and delayed psychomotor acquisition (see supplementary information for details). Finally, four 4-year-olds were unable to 
follow the task instructions or did not want to complete the experiment. We replaced one adult who exhibited unusual difficulty in handling the experimental setup and exhibited accuracy outside 2 s.d. of the adult sample in some experimental tasks.

Participants also performed a spatial memory span task, on which we report in the Supplementary Information.

The experiment was approved by the local ethics committee of Bielefeld University and was conducted in accordance with the Declaration of Helsinki. Adult participants and children's parents gave written informed consent prior to the experiment. Children gave oral consent after they had received all information prior to the experiment. Children received a small gift of about $10 €$ value for their participation. Participants of the adult control group received course credit.

\subsection{Experimental tasks}

We devised seven tasks in which participants had to move one or both hands in circles. Together, they address the three aspects of motor control we laid out in the introduction. The task setup mimicked turning wheels, that is, participants could move two handles in circles. We tested how well they could coordinate phase and speed of these circular movements.

\subsection{1 | Bimanual motor coordination}

Task 1 - Bimanual 1:1 Coordination Without Vision (Biman-NoVis): Participants had to move the two unseen hands symmetrically in six trials of $10 \mathrm{~s}$ duration each.

Task 2 - Bimanual 1:1 Coordination With Vision (Biman-Vis): Participants had to move the two hands symmetrically while receiving feedback about hand position in form of cursors on the screen above their hands. They performed six trials of $10 \mathrm{~s}$ duration each.

\subsection{2 | Unimanual motor coordination}

Task 3 - Match the Cursor: Unimanual Visual Coordination (Uniman-Vis): Participants had to coordinate their unimanual movement with an externally controlled visual signal. They saw a visual cursor moving along the same circular trajectory as the non-dominant hand in Biman-Vis. However, here the cursor was computer-controlled and the non-dominant hand rested on the lap. Participants had to symmetrically match the cursor position with their dominant hand. The position of their own hand, too, was visualized as a cursor. Thus, the task appeared visually identical to Biman-Vis. There were six trials of 20 s duration each. 
Task 4: Match the Other Hand: Unimanual Proprioceptive Coordination (Uniman-Prop): The robot passively moved the participant's non-dominant hand. Participants had to symmetrically match the passive movement of their non-dominant hand with their dominant hand. They were instructed to relax the passively guided arm and let the robot lead them without actively supporting the movement. No visual feedback of either hand's position was available. There were six trials of $20 \mathrm{~s}$ duration each.

\subsubsection{Sensory selectivity in bimanual motor coordination}

Task 5 - 2:3 Coordination With Veridical Hand Position Feedback (2:3-NoTransform): Participants had to concurrently perform three circles with their dominant, and two with their non-dominant hand. Hand position was indicated by visual cursors, as in Biman-Vis and Uniman-Vis. Accordingly, if the task was performed correctly, the two cursors did not move symmetrically. This kind of task is very difficult even for adults and constituted the baseline 2:3 coordination task. Participants performed six trials of $20 \mathrm{~s}$ duration each.

Task 6 - 2:3 Coordination With Transformed Feedback (2:3-Transform): Participants had to perform 2:3 coordination as in 2:3-NoTransform. However, cursor position did not reflect the hands' veridical location, but was transformed to appear symmetrical: For the dominant hand, the cursor completed one full circle on the display while participants performed 3 circles with their hand. For the nondominant hand, the cursor completed one circle while the hand circled twice. Accordingly, participants could correctly perform the task by maintaining a symmetrical visual feedback on the display. We explicitly explained this task feature to participants. They performed $3 \times 6$ trials of $20 \mathrm{~s}$ duration each. The higher trial number was introduced to allow learning, which we tested in 2:3Retention.

Task 7: 2:3 Coordination - Recall Without Feedback (2:3-Retention): Participants had to perform three circles with their dominant, and two with their non-dominant hand. Each trial began with transformed visual feedback just like 2:3-Transform. However, after 5 s, visual feedback was removed. Participants had to continue the 2:3 coordination, allowing us to assess their ability to perform the task feedback after having previously learned 2:3-Transform. Neither children nor adults exhibited any retention in

\section{3 | Task setup and procedure}

All tasks were implemented on the KINARM End-Point-Lab (BKIN Technologies Ltd, Kingston, Ontario, Canada), a robot that serves as a hand manipulandum for movements in a 2D horizontal plane in 
front of the user. Participants grab two handles whose position is continually measured at a sampling rate of $1000 \mathrm{~Hz}$. Mobility of the handles can be constrained by the robot. A monitor projects from above onto a mirror placed above the hands, so that visual information displayed on the mirror appears to be in the same plane as the hands.

Depending on their body height, participants were seated on a height-adjustable chair, or stood on an adjustable platform, so that their elbows were angled at $90^{\circ}$. Most children aged 7 and older were tall enough to be seated.

The overall setup was similar across all tasks. Hand movement was restricted via the Kinarm to movement along two circles of $10 \mathrm{~cm}$ diameter, one for each hand. The two circles were visualized in a common color and were separated by a straight vertical line that was styled as a fence. In its middle was a white fixation square. The circle centers were $10 \mathrm{~cm}$ away from the fixation point. On each trial, the hands started at the innermost circle positions, which were marked as blue, $1 \mathrm{~cm}$ diameter dots on the circles. We gave the tasks a child-friendly design and story line to support children's motivation to complete the experiment. Each child chose one of several stimulus themes that each featured four $2 \mathrm{~cm}$ diameter characters to represent hand position (see Fig. 1).

An opaque apron attached to the participant's neck prevented vision of the arms, and the display mirror was opaque, so that participants could not see their hands. This setup allowed us to fully control visual feedback about hand position via cursors on the screen.

In all tasks, a trial began with participants first moving their hand(s) to the starting position(s) (Fig. 1, blue dots). A beep of $150 \mathrm{~ms}$ duration instructed them to begin circling in the inward direction until a beep of $300 \mathrm{~ms}$ duration signaled the end of the trial. The eyes had to fixate the fixation square.

For all tasks, participants performed three practice trials during which we gave oral feedback on whether task performance complied with the instructions. If necessary, we added up to two additional practice trials. During experimental trials, we gave no feedback, unless if the direction of rotation was incorrect; in this case, the trial was repeated. The experimenter watched whether the child kept fixation; if not, the respective trial was repeated.

After every two trials, we displayed short sentences on the screen for $5 \mathrm{~s}$ to frame the experiment as a story and support children's motivation. These sentences playfully reflected the task instruction. For instance, one sentence of Biman-Vis was "While the dog is away, the cat steals its food. The dog runs after the cat"; 2:3-NoTransform included "The sheep and the dog are racing; but the dog is way faster", and 2:3-Transform and 2:3-Retention included "Remember that the animals want to run together". We read the sentences aloud for children who could not yet read. 
Participants were first acquainted with the Kinarm by playing a short game. They then performed the seven circling tasks and the memory span task in the order illustrated in Table 5. Participants always started with the bimanual coordination tasks, Biman-NoVis and Biman-Vis. We always devised these two tasks in this order because we wanted to avoid that performance without vision would benefit from experience of the visual task if it were done first. Then, UniMan-Vis, Uniman-Prop, 2:3NoTransform and the memory span task were conducted in randomized order by participants drawing pieces of paper in an envelope. The session ended with 2:3-Transform and 2:3-Retention. 2:3-Transform had to be conducted after 2:3-NoTransform because it potentially involved learning. In turn, 2:3-Retention tested learning of 2:3-Transform and, thus, had to be done last. All tasks were 3$5 \mathrm{~min}$ long, except for 2:3-Transform, which lasted $12 \mathrm{~min}$. Participants rested between tasks, and after every 6 trials in 2:3 Transform.

Not all participants performed all tasks. Table 5 lists the number of individuals per year of age and task. We lost some data due to equipment failure; this was especially prevalent for Uniman-Prop; thus, the low number of young participants is not due to younger children being unable to perform the task. However, some children, especially at younger ages, found it difficult to maintain motivation and to stay focused; some young children did not understand the 2:3 tasks; thus, for those tasks, low participant numbers are due to task difficulty, not technical problems.

\section{4 | Data processing and analysis}

We analyzed data in Matlab (version: R2015aSP1). Each hand's position time series was filtered using a low pass Butterworth filter with a cut-off frequency of $10 \mathrm{~Hz}$. We extracted three measures: signed and unsigned phase difference, and percentage of adequate coordination.

Signed and unsigned phase difference. We coded individual positions as the angle between the circles' innermost position (which was also the starting position) and location on the circle along movement direction. Thus, when the hands were at the lowest position, their phase angle was $90^{\circ}$, at the outermost position it was $180^{\circ}$ etc. Because participants performed continuously for an extended duration, they performed several circles. In the bimanual tasks, we computed the phase angle of the dominant minus the non-dominant hand. In the unimanual tasks, we computed the phase angle difference between the moving hand and the position of the leading signal, that is, the cursor or the passively moved hand. In the 2:3 tasks we transformed each hand's position to the transformed cursor position. This involved dividing the dominant hand's phase angle by 3 , and the non-dominant hand's phase angle by 2 . These two transformed locations would be in perfect phase if participants 
performed the 2:3 task correctly. We, thus, report the difference between transformed phase angles for these tasks.

We analyzed unsigned and signed error. Signed error is the absolute phase angle difference between the two entities that had to be coordinated - the two hands in bimanual tasks; the visual cursor or the passively guided hand and the active hand in unimanual tasks, and the two transformed locations in the 2:3 tasks. For any given sampling point, a positive (negative) error indicates that the dominant/active hand leads (lags) the other hand or cursor. Because the phase difference is unsigned, both leading and lagging increase variable error in the same manner. In addition, we report the average signed error which signifies how much the dominant hand leads or lags on average; here, positive and negative error average out.

Percentage of adequate coordination. For comparison with some previous studies, we report adequate performance as a maximal deviation of $+/-30^{\circ}$ from the correct phase difference as an additional measure in the supplementary information (Mechsner et al., 2001; Mechsner, Stenneken, Cole, Aschersleben, \& Prinz, 2007). This measure is similar to the proportion of time-on-task used by some authors (e.g. Bingham, Snapp-Childs, \& Zhu, 2018), and renders comparable conclusions to those obtained with raw angle measures (Brandes et al., 2017).

Analysis strategy. We separately analyzed Tasks 1-4 (symmetrical tasks) and 5-7 (2:3 tasks). To assess changes in performance across development, we computed two models for each of the two sets of tasks: one in which age in months was used as a continuous predictor, and one in which we contrasted age groups, pooled in years. In a second type of analysis we aimed to assess whether there were developmental dependencies across tasks. We compared performance between each pair of tasks while partialing out age to eliminate trivial correlation due to general improvement over age. Adults were only included in the analysis contrasting age groups, but not in the analysis with continuous age in months, nor in the correlations.

Models of unsigned difference with a continuous age variable. We statistically analyzed data in $R$ ( $R$ Core Team, 2018; R: 3.5.1; R Studio: 1.1.456). We implemented multilevel Bayesian modeling with the $\mathrm{R}$ package brms (Bürkner, 2017). The dependent variable was the averaged phase angle difference in degrees per full circle of the dominant hand, rendering multiple observations per subject in the different experimental tasks, that is, completed circles within trial and multiple trials per subject. This analysis strategy captured performance variability within trials, rather than averaging together entire 10 or $20 \mathrm{~s}$ chunks into a single measurement value, while keeping model fitting computationally tractable. The independent predictors comprised the within-subject, categorical variable Task (Task 1-4: Biman-NoVis, Biman-Vis, Uniman-Vis and Uniman-Prop; Task 5-7: 
2:3-NoTransform, 2:3-Transform and 2:3-Retention), the between-subject, continuous variable Age (in months), and the interaction between Task and Age. The model was defined as: Phase_Difference $\sim$ Task * Age $+(1 \mid$ subject $)+(1 \mid$ trial $)$, thus allowing random effects per subject and trial. The model was run with one reference condition. We calculated contrasts that reflected comparisons of interest by comparing the factor sums of the involved condition estimates; for instance, to compute the estimate of a non-reference variable, all main and interaction estimates involving this condition are added the intercept. An alternative strategy is to re-run models with the condition of interest as reference condition; we obtained equivalent results with both strategies.

Models of unsigned difference with age groups. In these models, one for Tasks 1-4 and one for Tasks 5-7, the predictor Age was grouped by year as a categorical variable. The model was defined similarly to the previous models as: Phase_Difference Task * Group $+(1 \mid$ subject $)+(1 \mid$ trial $)$. We used these models to test at which age (in years) children performed similarly as adults, and to contrast performance between Tasks within a given age group.

Models of signed difference with age coded as continuous and categorical variable. We also fitted the four models described so far with signed phase angle difference between the two hands as dependent variable.

All described models were fitted using a Gaussian likelihood and the brms package default weaklyinformative, improper, flat priors for population-level (“fixed") effects. The estimation of parameters' posterior distribution were obtained by Hamiltonian Monte-Carlo sampling with 4 chains, 1,000 sample warmup, and 11,000 iterations, and checked visually for convergence (high ESS and Rhat $\approx 1$ ). For each main effect and interaction, we report the estimate of the mean and its $95 \%$ high-density interval (HDI), that is, the interval that covers $95 \%$ of the posterior distribution. We used the $R$ package sjstats (Lüdecke, 2018) for inference on the presence of statistical effects, testing for each factor whether the HDI excluded a region of practical equivalence (ROPE) of effect sizes of 0.1 around 0 (Kruschke, 2011, 2018). This approach is conservative, because it requires not only that the HDI exclude 0, but in addition that no overlap exist with the ROPE interval (Kruschke, 2011). For models with a continuous predictor Age, we standardized the posterior distribution of this covariate (and respective interactions) by multiplying the covariate and respective interaction estimates (and their respective HDIs) by the standard deviation of subjects' age.

Relationship between tasks beyond age. We correlated unsigned phase angle between pairs of tasks, but removed the effect of age using Bayesian partial correlations as implemented in the R package 
bioRxiv preprint doi: https://doi.org/10.1101/601435; this version posted July 18,2019 . The copyright holder for this preprint (which was not certified by peer review) is the author/funder. All rights reserved. No reuse allowed without permission.

Development of uni- and bimanual coordination

page 28

BayesMed (Wetzels \& Wagenmakers, 2012) using a default Jeffrey's-Zellner-Siow prior set-up (Nuijten, Wetzels, Matzke, Dolan, \& Wagenmakers, 2015). This analysis strategy ensured that any relationships revealed by correlation analysis cannot simply be due to unspecific improvement across all tasks with age. 


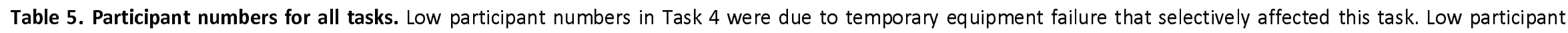

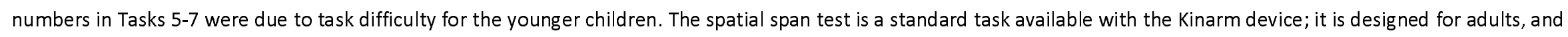
many younger children could not reach all positions involved in the task. We therefore did not test children who were too small for this task.

\begin{tabular}{|c|c|c|c|c|c|c|c|c|c|c|}
\hline $\begin{array}{c}\text { Age Group } \\
\text { Number of included participants }\end{array}$ & $\begin{array}{l}4 \text { yo } \\
n=14\end{array}$ & $\begin{array}{c}5 \text { yo } \\
n=12\end{array}$ & $\begin{array}{c}6 \text { yo } \\
n=12\end{array}$ & $\begin{array}{l}7 \text { yo } \\
n=12\end{array}$ & $\begin{array}{c}8 \text { yo } \\
n=12\end{array}$ & $\begin{array}{l}9 \text { yo } \\
n=16\end{array}$ & $\begin{array}{l}10 \text { yo } \\
n=18\end{array}$ & $\begin{array}{l}11 \text { yo } \\
n=12\end{array}$ & $\begin{array}{l}12 \text { yo } \\
n=12\end{array}$ & $\begin{array}{l}\text { Adults } \\
n=12\end{array}$ \\
\hline $\begin{array}{l}\text { Control task: } \\
\text { Spatial Span }\end{array}$ & 1 & 7 & 11 & 11 & 12 & 15 & 18 & 12 & 12 & 12 \\
\hline $\begin{array}{c}\text { Task } 1 \text { - Bimanual 1:1 Coordination } \\
\text { Without Vision - Biman-NoVis }\end{array}$ & 14 & 12 & 12 & 12 & 12 & 16 & 18 & 12 & 12 & 12 \\
\hline $\begin{array}{c}\text { Task } 2 \text { - Bimanual 1:1 Coordination } \\
\text { With Vision - Biman-Vis }\end{array}$ & 14 & 12 & 12 & 12 & 12 & 16 & 18 & 12 & 12 & 12 \\
\hline $\begin{array}{c}\text { Task } 3 \text { - Match the Cursor: Unimanual Visual } \\
\text { Coordination - Uniman-Vis }\end{array}$ & 14 & 12 & 12 & 12 & 11 & 16 & 18 & 12 & 12 & 12 \\
\hline $\begin{array}{l}\text { Task } 4 \text { - Match the Other Hand: Unimanual } \\
\text { Proprioceptive Coordination - Uniman-Prop }\end{array}$ & 1 & 2 & 3 & 5 & 4 & 7 & 6 & 12 & 12 & 12 \\
\hline & & & & & & & & & & \\
\hline $\begin{array}{c}\text { Task } 5-2: 3 \text { Coordination } \\
\text { With Veridical Hand Position Feedback - 2:3- } \\
\text { NoTransform }\end{array}$ & 6 & 3 & 11 & 12 & 11 & 16 & 18 & 12 & 12 & 12 \\
\hline $\begin{array}{c}\text { Task 6-2:3 Coordination } \\
\text { With Transformed Feedback - 2:3-Transform }\end{array}$ & 5 & 3 & 11 & 12 & 12 & 16 & 18 & 12 & 12 & 12 \\
\hline $\begin{array}{c}\text { Task 7 - 2:3 Coordination - } \\
\text { Recall Without Feedback - 2:3-Retention }\end{array}$ & 3 & 1 & 7 & 11 & 11 & 16 & 17 & 12 & 12 & 12 \\
\hline
\end{tabular}




\section{DATA AND SOFTWARE AVAILABILITY}

All data and code used in this study are available on the website of the Open Science Framework (OSF) and can be accessed at https://osf.io/x9u27/

\section{REFERENCES}

Adolph, K. E., \& Franchak, J. M. (2017). The development of motor behavior. Wiley Interdisciplinary Reviews: Cognitive Science, 8(1-2), n/a-n/a. https://doi.org/10.1002/wcs.1430

Adolph, K. E., \& Hoch, J. E. (2019). Motor Development: Embodied, Embedded, Enculturated, and Enabling. Annual Review of Psychology, 70(1), null. https://doi.org/10.1146/annurev-psych010418-102836

Adolph, K. E., Hoch, J. E., \& Cole, W. G. (2018). Development (of Walking): 15 Suggestions. Trends in Cognitive Sciences, 22(8), 699-711. https://doi.org/10.1016/j.tics.2018.05.010

Assaiante, C., Barlaam, F., Cignetti, F., \& Vaugoyeau, M. (2014). Body schema building during childhood and adolescence: a neurosensory approach. Neurophysiologie Clinique $=$ Clinical Neurophysiology, 44(1), 3-12. https://doi.org/10.1016/j.neucli.2013.10.125

Badan, M., Hauert, C. A., \& Mounoud, P. (2000). Sequential pointing in children and adults. Journal of Experimental Child Psychology, 75(1), 43-69. https://doi.org/10.1006/jecp.1999.2522

Bingham, G. P. (2004). A Perceptually Driven Dynamical Model of Bimanual Rhythmic Movement (and Phase Perception). Ecological Psychology, 16(1), 45-53.

https://doi.org/10.1207/s15326969eco1601_6

Bingham, G. P., Snapp-Childs, W., \& Zhu, Q. (2018). Information about relative phase in bimanual coordination is modality specific (not amodal), but kinesthesis and vision can teach one another. Human Movement Science, 60, 98-106.

https://doi.org/10.1016/j.humov.2018.05.010

Brandes, J., Rezvani, F., \& Heed, T. (2016). Visual guidance of bimanual coordination relies on movement direction (No. biorxiv;063404v1). Retrieved from http://biorxiv.org/lookup/doi/10.1101/063404

Brandes, J., Rezvani, F., \& Heed, T. (2017). Abstract spatial, but not body-related, visual information guides bimanual coordination. Scientific Reports, 7(1), 16732. https://doi.org/10.1038/s41598-017-16860-x

Buchanan, J. J., \& Wang, C. (2012). Overcoming the guidance effect in motor skill learning: feedback all the time can be beneficial. Experimental Brain Research, 219(2), 305-320. https://doi.org/10.1007/s00221-012-3092-x

Buekers, M. J., Bogaerts, H. P., Swinnen, S. P., \& Helsen, W. F. (2000). The synchronization of human arm movements to external events. Neuroscience Letters, 290(3), 181-184. https://doi.org/10.1016/S0304-3940(00)01350-1

Bürkner, P.-C. (2017). brms: An R Package for Bayesian Multilevel Models Using Stan. Journal of Statistical Software, 80(1), 1-28. https://doi.org/10.18637/jss.v080.i01 
Burr, D., \& Gori, M. (2012). Multisensory Integration Develops Late in Humans. In M. M. Murray \& M. T. Wallace (Eds.), The Neural Bases of Multisensory Processes. Retrieved from http://www.ncbi.nlm.nih.gov/books/NBK92864/

Chiou, S.-C., \& Chang, E. C. (2016). Bimanual Coordination Learning with Different Augmented Feedback Modalities and Information Types. PLOS ONE, 11(2), e0149221. https://doi.org/10.1371/journal.pone.0149221

Cohen, L. (1971). Synchronous Bimanual Movements Performed by Homologous and NonHomologous Muscles. Perceptual and Motor Skills, 32(2), 639-644. https://doi.org/10.2466/pms.1971.32.2.639

Contreras-Vidal, J. L. (2006). Development of forward models for hand localization and movement control in 6- to 10-year-old children. Human Movement Science, 25(4-5), 634-645. https://doi.org/10.1016/j.humov.2006.07.006

Contreras-Vidal, J. L., Bo, J., Boudreau, J. P., \& Clark, J. E. (2005). Development of visuomotor representations for hand movement in young children. Experimental Brain Research, 162(2), 155-164. https://doi.org/10.1007/s00221-004-2123-7

Daseking, M., \& Petermann, F. (2013). Verhaltensinventar zur Beurteilung exekutiver Funktionen für das Kindergartenalter, BRIEF-P. Deutschsprachige Adaptation des Behavior Rating Inventory of Executive Function for Preschoolers.

de Boer, B. J., Peper, C. E., \& Beek, P. J. (2012). Development of temporal and spatial bimanual coordination during childhood. Motor Control, 16(4), 537-559.

Drechsler, R., \& Steinhausen, H.-C. (2013). Verhaltensinventar zur Beurteilung exekutiver Funktionen, BRIEF. Deutschsprachige Adaptation des Behavior Rating Inventory of Executive Function.

Fagard, J., Hardy-Léger, I., Kervella, C., \& Marks, A. (2001). Changes in interhemispheric transfer rate and the development of bimanual coordination during childhood. Journal of Experimental Child Psychology, 80(1), 1-22. https://doi.org/10.1006/jecp.2000.2623

Forssberg, H., Eliasson, A. C., Kinoshita, H., Johansson, R. S., \& Westling, G. (1991). Development of human precision grip. I: Basic coordination of force. Experimental Brain Research, 85(2), 451457.

Giedd, J. N., Rumsey, J. M., Castellanos, F. X., Rajapakse, J. C., Kaysen, D., Vaituzis, A. C., ... Rapoport, J. L. (1996). A quantitative MRI study of the corpus callosum in children and adolescents. Brain Research. Developmental Brain Research, 91(2), 274-280.

Gooijers, J., \& Swinnen, S. P. (2014). Interactions between brain structure and behavior: The corpus callosum and bimanual coordination. Neuroscience \& Biobehavioral Reviews, 43, 1-19. https://doi.org/10.1016/j.neubiorev.2014.03.008

Gori, M. (2015). Multisensory Integration and Calibration in Children and Adults with and without Sensory and Motor Disabilities. Multisensory Research, 28(1-2), 71-99.

Hamilton, A. F. de C., Southgate, V., \& Hill, E. L. (2016). The development of action cognition. In A. K. Engel, K. J. Friston, \& D. Kragic (Eds.), The Pragmatic Turn: Toward Action-Oriented Views in Cognitive Science (pp. 35-47). Retrieved from https://mitpress.mit.edu/books/pragmaticturn 
Hay, L. (1978). Accuracy of children on an open-loop pointing task. Perceptual and Motor Skills, 47/3 Pt 2), 1079-1082. https://doi.org/10.2466/pms.1978.47.3f.1079

Hay, L., Bard, C., Ferrel, C., Olivier, I., \& Fleury, M. (2005). Role of proprioceptive information in movement programming and control in 5 to 11 -year old children. Human Movement Science, 24(2), 139-154. https://doi.org/10.1016/j.humov.2005.05.002

Hay, L., Fleury, M., Bard, C., \& Teasdale, N. (1994). Resolving power of the perceptual- and sensorimotor systems in 6- to 10-year-old children. Journal of Motor Behavior, 26(1), 36-42. https://doi.org/10.1080/00222895.1994.9941659

Heed, T., \& Röder, B. (2014). Motor coordination uses external spatial coordinates independent of developmental vision. Cognition, 132(1), 1-15.

https://doi.org/10.1016/j.cognition.2014.03.005

Hove, M. J., Spivey, M. J., \& Krumhansl, C. L. (2010). Compatibility of motion facilitates visuomotor synchronization. Journal of Experimental Psychology. Human Perception and Performance, 36(6), 1525-1534. https://doi.org/10.1037/a0019059

Jeeves, M. A., Silver, P. H., \& Milne, A. B. (1988). Role of the corpus callosum in the development of a bimanual motor skill. Developmental Neuropsychology, 4(4), 305-323. https://doi.org/10.1080/87565648809540415

Kagerer, F. A., \& Clark, J. E. (2014). Development of interactions between sensorimotor representations in school-aged children. Human Movement Science, 34, 164-177. https://doi.org/10.1016/j.humov.2014.02.001

Kelso, J. A. S. (1984). Phase transitions and critical behavior in human bimanual coordination. The American Journal of Physiology, 246(6 Pt 2), R1000-1004. https://doi.org/10.1152/ajpregu.1984.246.6.R1000

Kelso, J. A. S., Fink, P. W., DeLaplain, C. R., \& Carson, R. G. (2001). Haptic information stabilizes and destabilizes coordination dynamics. Proceedings of the Royal Society of London. Series B: Biological Sciences, 268(1472), 1207-1213. https://doi.org/10.1098/rspb.2001.1620

Kelso, J. A. S., Scholz, J. P., \& Schöner, G. (1986). Nonequilibrium phase transitions in coordinated biological motion: critical fluctuations. Physics Letters A, 118(6), 279-284. https://doi.org/10.1016/0375-9601(86)90359-2

Kennerley, S. W., Diedrichsen, J., Hazeltine, E., Semjen, A., \& Ivry, R. B. (2002). Callosotomy patients exhibit temporal uncoupling during continuous bimanual movements. Nature Neuroscience, 5(4), 376-381. https://doi.org/10.1038/nn822

King, B. R., Pangelinan, M. M., Kagerer, F. A., \& Clark, J. E. (2010). Improvements in proprioceptive functioning influence multisensory-motor integration in 7- to 13-year-old children. Neuroscience Letters, 483(1), 36-40. https://doi.org/10.1016/j.neulet.2010.07.056

Konczak, J., Jansen-Osmann, P., \& Kalveram, K.-T. (2003). Development of force adaptation during childhood. Journal of Motor Behavior, 35(1), 41-52. https://doi.org/10.1080/00222890309602120

Kovacs, A. J., Buchanan, J. J., \& Shea, C. H. (2009). Bimanual 1:1 with 90 degrees continuous relative phase: difficult or easy! Experimental Brain Research, 193(1), 129-136. https://doi.org/10.1007/s00221-008-1676-2 
Kovacs, A. J., \& Shea, C. H. (2011). The learning of $90^{\circ}$ continuous relative phase with and without Lissajous feedback: external and internally generated bimanual coordination. Acta Psychologica, 136(3), 311-320. https://doi.org/10.1016/j.actpsy.2010.12.004

Kruschke, J. K. (2011). Bayesian Assessment of Null Values Via Parameter Estimation and Model Comparison. Perspectives on Psychological Science, 6(3), 299-312. https://doi.org/10.1177/1745691611406925

Kruschke, J. K. (2018). Rejecting or Accepting Parameter Values in Bayesian Estimation. Advances in Methods and Practices in Psychological Science, 1(2), 270-280. https://doi.org/10.1177/2515245918771304

Lantero, D. A., \& Ringenbach, S. D. R. (2007). Developmental differences in the use of visual information during a continuous bimanual coordination task. Journal of Motor Behavior, 39(2), 139-155. https://doi.org/10.3200/JMBR.39.2.139-157

Leinen, P., Vieluf, S., Kennedy, D., Aschersleben, G., Shea, C. H., \& Panzer, S. (2016). Life span changes: Performing a continuous 1:2 bimanual coordination task. Human Movement Science, 46, 209-220. https://doi.org/10.1016/j.humov.2016.01.004

Lüdecke, D. (2018). sjstats: Statistical Functions for Regression Models. https://doi.org/10.5281/zenodo.1489175

Mechsner, F., Kerzel, D., Knoblich, G., \& Prinz, W. (2001). Perceptual basis of bimanual coordination. Nature, 414(6859), 69-73. https://doi.org/10.1038/35102060

Mechsner, F., Stenneken, P., Cole, J., Aschersleben, G., \& Prinz, W. (2007). Bimanual circling in deafferented patients: evidence for a role of visual forward models. Journal of Neuropsychology, 1(Pt 2), 259-282.

Muetzel, R. L., Collins, P. F., Mueller, B. A., M Schissel, A., Lim, K. O., \& Luciana, M. (2008). The development of corpus callosum microstructure and associations with bimanual task performance in healthy adolescents. Neurolmage, 39(4), 1918-1925. https://doi.org/10.1016/j.neuroimage.2007.10.018

Nuijten, M. B., Wetzels, R., Matzke, D., Dolan, C. V., \& Wagenmakers, E.-J. (2015). A default Bayesian hypothesis test for mediation. Behavior Research Methods, 47(1), 85-97. https://doi.org/10.3758/s13428-014-0470-2

Oliveira, F. T. P., \& Ivry, R. B. (2008). The Representation of Action: Insights From Bimanual Coordination. Current Directions in Psychological Science, 17(2), 130-135. https://doi.org/10.1111/j.1467-8721.2008.00562.x

Oswald, W. D., \& Roth, E. (1978). Der Zahlen-Verbindungs-Test (ZVT). Göttingen, Germany: Hogrefe.

Pellizzer, G., \& Hauert, C.-A. (1996). Visuo-manual aiming movements in 6-to 10-year-old children: evidence for an asymmetric and asynchronous development of information processes. Brain and Cognition, 30(2), 175-193.

R Core Team. (2018). $R$ : A language and environment for statistical computing. $R$ Foundation for Statistical Computing, Vienna, Austria. URL https://www.R-project.org/. 
Repp, B. H., \& Steinman, S. R. (2010). Simultaneous Event-Based and Emergent Timing: Synchronization, Continuation, and Phase Correction. Journal of Motor Behavior, 42(2), 111126. https://doi.org/10.1080/00222890903566418

Riek, S., \& Woolley, D. (2005). Hierarchical organisation of neuro-anatomical constraints in interlimb coordination. Human Movement Science, 24(5), 798-814. https://doi.org/10.1016/j.humov.2005.10.002

Robertson, S. D. (2001). Development of bimanual skill: the search for stable patterns of coordination. Journal of Motor Behavior, 33(2), 114-126. https://doi.org/10.1080/00222890109603144

Roy, A. C., Curie, A., Nazir, T., Paulignan, Y., des Portes, V., Fourneret, P., \& Deprez, V. (2013). Syntax at hand: common syntactic structures for actions and language. PloS One, 8(8), e72677. https://doi.org/10.1371/journal.pone.0072677

Shea, C. H., Buchanan, J. J., \& Kennedy, D. M. (2016). Perception and action influences on discrete and reciprocal bimanual coordination. Psychonomic Bulletin \& Review, 23(2), 361-386. https://doi.org/10.3758/s13423-015-0915-3

Sigrist, R., Rauter, G., Riener, R., \& Wolf, P. (2013). Augmented visual, auditory, haptic, and multimodal feedback in motor learning: a review. Psychonomic Bulletin \& Review, 20(1), 2153. https://doi.org/10.3758/s13423-012-0333-8

Smyth, M. M., Katamba, J., \& Peacock, K. A. (2004). Development of prehension between 5 and 10 years of age: distance scaling, grip aperture, and sight of the hand. Journal of Motor Behavior, 36(1), 91-103. https://doi.org/10.3200/JMBR.36.1.91-103

Smyth, M. M., Peacock, K. A., \& Katamba, J. (2004). The role of sight of the hand in the development of prehension in childhood. The Quarterly Journal of Experimental Psychology. A, Human Experimental Psychology, 57(2), 269-296. https://doi.org/10.1080/02724980343000215

Spencer, R. M. C., \& Ivry, R. B. (2007). The temporal representation of in-phase and anti-phase movements. Human Movement Science, 26(2), 226-234. https://doi.org/10.1016/j.humov.2007.01.002

Spencer, R. M. C., Ivry, R. B., Cattaert, D., \& Semjen, A. (2005). Bimanual coordination during rhythmic movements in the absence of somatosensory feedback. Journal of Neurophysiology, 94(4), 2901-2910. https://doi.org/10.1152/jn.00363.2005

Spencer, R. M. C., Semjen, A., Yang, S., \& Ivry, R. B. (2006). An event-based account of coordination stability. Psychonomic Bulletin \& Review, 13(4), 702-710.

Swinnen, S. P., Jardin, K., Verschueren, S., Meulenbroek, R., Franz, L., Dounskaia, N., \& Walter, C. B. (1998). Exploring interlimb constraints during bimanual graphic performance: effects of muscle grouping and direction. Behavioural Brain Research, 90(1), 79-87.

Tanaka-Arakawa, M. M., Matsui, M., Tanaka, C., Uematsu, A., Uda, S., Miura, K., ... Noguchi, K. (2015). Developmental changes in the corpus callosum from infancy to early adulthood: a structural magnetic resonance imaging study. PloS One, 10(3), e0118760.

https://doi.org/10.1371/journal.pone.0118760 
Temprado, J. J., Swinnen, S. P., Carson, R. G., Tourment, A., \& Laurent, M. (2003). Interaction of directional, neuromuscular and egocentric constraints on the stability of preferred bimanual coordination patterns. Human Movement Science, 22(3), 339-363.

Tomatsu, S., \& Ohtsuki, T. (2005). The effect of visual transformation on bimanual circling movement. Experimental Brain Research, 166(2), 277-286. https://doi.org/10.1007/s00221-005-2363-1

Uda, S., Matsui, M., Tanaka, C., Uematsu, A., Miura, K., Kawana, I., \& Noguchi, K. (2015). Normal development of human brain white matter from infancy to early adulthood: a diffusion tensor imaging study. Developmental Neuroscience, 37(2), 182-194. https://doi.org/10.1159/000373885

Viel, S., Vaugoyeau, M., \& Assaiante, C. (2009). Adolescence: a transient period of proprioceptive neglect in sensory integration of postural control. Motor Control, 13(1), 25-42.

Wetzels, R., \& Wagenmakers, E.-J. (2012). A default Bayesian hypothesis test for correlations and partial correlations. Psychonomic Bulletin \& Review, 19(6), 1057-1064.

https://doi.org/10.3758/s13423-012-0295-x

Wilson, P. H., \& Hyde, C. E. (2013). The development of rapid online control in children aged 612 years: Reaching performance. Human Movement Science. https://doi.org/10.1016/j.humov.2013.02.008

Wing, A. M., Doumas, M., \& Welchman, A. E. (2010). Combining multisensory temporal information for movement synchronisation. Experimental Brain Research, 200(3), 277-282. https://doi.org/10.1007/s00221-009-2134-5 